\title{
On the origin of ultra high energy cosmic rays: subluminal and superluminal relativistic shocks
}

\author{
A. Meli ${ }^{1,2,3}$, J. K. Becker ${ }^{2,4}$, and J. J. Quenby ${ }^{5}$ \\ 1 Department of Physics, National University of Athens, Panepistimiopolis Zografos 15783, Greece \\ 2 Institut für Physik, Universität Dortmund, 44221 Dortmund, Germany \\ 3 Erlangen Center for Astroparticle Physics, Universität Erlangen-Nürnberg, Germany \\ e-mail: athina.meli@physik.uni-erlangen.de \\ ${ }^{4}$ Institutionen för Fysik, Göteborgs Universitet, 41296 Göteborg, Sweden \\ e-mail: julia.becker@physics.gu.se \\ 5 High Energy Physics Group, Blackett Laboratory, Imperial College London, Prince Consort Road, London SW7 2BZ, UK \\ e-mail: j.quenby@imperial.ac.uk
}

Received 15 September 2007 / Accepted 1 October 2008

\section{ABSTRACT}

\begin{abstract}
Aims. The flux of ultra high energy cosmic rays (UHECRs) at $E>10^{18.5} \mathrm{eV}$ is believed to arise in plasma shock environments in extragalactic sources. In this paper, we present a systematic study of cosmic ray $(\mathrm{CR})$ particle acceleration by relativistic shocks, in particular concerning the dependence on bulk Lorentz factor and the angle between the magnetic field and the shock flow. The contribution to the observed diffuse CR spectrum provided by the accelerated particles is discussed.

Methods. For the first time, Monte Carlo simulations for super- and subluminal shocks are extended to boost factors up to $\Gamma=1000$ and systematically compared. The source spectra derived are translated into the expected diffuse proton flux from astrophysical sources by folding the spectra with the spatial distribution of active galactic nuclei (AGN) and gamma ray bursts (GRBs). Results of these predictions are compared with UHECR data.

Results. While superluminal shocks are shown to be inefficient at providing acceleration to the highest energies $\left(E>10^{18.5} \mathrm{eV}\right)$, subluminal shocks may provide particles up to $10^{21} \mathrm{eV}$, limited only by the Hillas-criterion. In the subluminal case, we find that mildly-relativistic shocks, thought to occur in jets of $\mathrm{AGN}(\Gamma \sim 10-30)$, yield energy spectra of $\mathrm{d} N / \mathrm{d} E \sim E^{-2}$. Highly relativistic shocks expected in GRBs $(100<\Gamma<1000)$, on the other hand, produce spectra as flat as $\sim E^{-1.0}$ above $10^{9.5} \mathrm{GeV}$. The model results are compared with the measured flux of CRs at the highest energies and it is shown that, while AGN spectra provide an excellent fit, GRB spectra are too flat to explain the observed flux. The first evidence of a correlation between the CR flux above $5.7 \times 10^{10} \mathrm{GeV}$ and the distribution of AGN provided by Auger are explained by our model. Although GRBs are excluded as the principle origin of UHECRs, neutrino production is expected in these sources either in mildly or highly relativistic shocks. In particular, superluminal shocks in GRBs may be observable via neutrino and photon fluxes, rather than as protons.
\end{abstract}

Key words. acceleration of particles - shock waves - waves - magnetic fields - scattering

\section{Introduction}

The observation of the energy spectrum of ultra high energy cosmic rays (UHECRs) indicates the presence of an extragalactic component at $E>10^{18.5} \mathrm{eV}$. Active galactic nuclei (AGN) and gamma ray bursts (GRBs) seem to be the two most promising source candidates for the production of charged cosmic rays (CRs) in this energy range. Work in the late 1970s by a number of authors, e.g. Krymskii (1977), Bell (1978a), Bell (1978b), based their ideas on the original Fermi acceleration mechanism of Fermi (1949), Fermi (1954), establishing the basic mechanism of particle diffusive acceleration in non-relativistic shocks. In this mechanism, individual particles are accelerated in a collisionless magnetised plasma by scattering off magnetic irregularities to recross a shock front many times. Since then, considerable analytical and numerical investigations have been performed, but questions remain concerning details of the acceleration mechanism at highly relativistic shock speeds.

In this work we present a series of Monte Carlo simulations with the aim of providing a more refined determination of the possible accelerated particle spectra that can result. All shocks under investigation are taken to be oblique, so that the magnetic field is neither aligned with nor strictly perpendicular to the shock front normal. Equivalent parallel shock simulations were provided in Meli \& Quenby (2003a). The calculations are performed in models of the relativistic shock environments believed to occur in AGN and GRB jets. We describe the very highenergy CR spectra resulting from the above candidate sources, and mention possible neutrino and photon emission. Previous work by Meli \& Quenby (2003a,b), studying subluminal and superluminal shocks did not establish systematic relationships between spectral slope, shock inclination angle, shock velocity and particle scattering model topics that will be considered here. These past investigations employed both the large-angle scattering model as traditionally used in many plasma transport studies and also the pitch angle diffusion model, but only for the extreme limiting case of pitch angle changes of $\delta \theta \leq 1 / \Gamma$, where $\theta$ is the angle of the particle velocity vector to the magnetic field direction and $\Gamma$ the Lorentz factor of the plasma flow. In the present work we allow pitch angle scattering to vary between $1 / \Gamma \leq \delta \theta \leq 10 / \Gamma$ to both allow for a more turbulent astrophysical magnetic field and to introduce a greater stochastic element 
into the scattering. We establish the spectral index dependence on the $\Gamma$ of the shock. The shock obliquity is varied while using a series of high velocity plasma flows, ranging from $\Gamma=10-1000$. The contribution to the observed diffuse CR spectrum provided by the high energy CR particles emerging from AGN and GRBs is discussed, based upon the simulated spectra.

In Sect. 2, details of the simulation are given. Section 3 presents the resulting spectra emphasising the dependence of the spectra on the Lorentz factor of the relativistic flow. The calculated spectra are used to estimate a possible contribution of AGN and GRBs to the observed diffuse spectrum of charged CRs. The implications for high energy neutrino and photon emission is discussed in Sect. 4. Finally, in Sect. 5 the results are summarised.

\subsection{Source candidates for relativistic shocks}

Based upon the observed energy release rate of gamma-rays in GRBs, the energy available for UHECR production in GRBs yields an estimated input rate of $2 \times 10^{44} \mathrm{erg} / \mathrm{Mpc}^{3} / \mathrm{yr}$. This energy budget corresponds to the energy release rate required for UHECR of $>10^{18.5} \mathrm{eV}$ and is based on an assumed star formation rate (SFR) history, e.g. Vietri (1995), Waxman (2000), with about $1 \mathrm{burst} / \mathrm{Gpc}^{3} / \mathrm{yr}$ at $z=0$. With a typical luminosity of AGN, $L \sim 10^{42}-10^{47} \mathrm{erg} / \mathrm{s}$, it is also possible to explain the UHECR flux as originating in AGN under the assumption that these objects follow the SFR. For example, Moran et al. (2001) find an average luminosity from an estimated $95 \mathrm{X}$-ray active AGN within $60 \mathrm{Mpc}$ of $4.8 \times 10^{44} \mathrm{erg} / \mathrm{s}$, this distance being the $\mathrm{CR}$ absorption horizon at about $2 \times 10^{20} \mathrm{eV}$. Within the regions of local space accessible to CR diffusion, the energy supply over a Hubble time is $6.6 \times 10^{-16} \mathrm{erg} / \mathrm{cm}^{3}$. By comparison the GRB supply is $6.7 \times 10^{-20} \mathrm{erg} / \mathrm{cm}^{3}$. With GRBs as the most luminous transient objects in the sky and AGN as the most luminous permanent ones, these two classes of sources constitute the most likely candidates for the acceleration of UHECR. They both fulfill the source criteria defined by Hillas (1984) as will be discussed later.

Interpretation of data on the electron synchrotron radiation observed in the radio regime suggests the presence of mildlyrelativistic shocks with boost factors $\Gamma \approx 10-30$ in the jets of AGN, see Biermann \& Strittmatter (1987), Falcke et al. (1995). The photon spectrum of AGN is broadband, ranging from radio up to $\mathrm{TeV}$ emission when the sources are optically thin. Assuming that hadrons are accelerated along with the electrons in the jet, AGN thus appear as good candidates to provide at least a significant fraction of the extragalactic component of the CR flux.

The prompt GRB photon spectra have a break energy which shifts with the boost factor. Therefore, the observation of the break energy can help determining the boost factor for individual bursts. In the case of a break at $E_{\gamma}>500 \mathrm{keV}$, the requirement that the optical depth in the burst is about unity determines $\Gamma$. For break energies $E_{\gamma}<500 \mathrm{keV}$, the synchrotron spectral break indicates $\Gamma$. Boost factors in the range $100<\Gamma<1000$ are found, see Halzen \& Hooper (2002) and references therein. However, mildly relativistic, internal, reverse shocks may also occur when viewed in the GRB plasma flow reference frame. Based on these boost values, it is estimated that protons maybe accelerated up to $\sim 10^{21} \mathrm{eV}$, see especially Waxman (2000). Calculating the total amount of electromagnetic energy released by GRBs then leads to the suggestion that the CR spectrum above the ankle $\left(E>10^{18.5} \mathrm{eV}\right)$ can be accounted for by GRBs, e.g. Vietri (1995).

\subsection{Maximum energy}

A basic physical limitation to the maximum energy of the accelerated particles is the product of the size of the acceleration region and the magnetic field present within this region. Parker (1958) first discussed limitations to the CR energy for a comprehensive set of acceleration mechanisms and later on Hillas (1984) extended the CR energy limit argument to several classes of astrophysical sources. He equated the time to diffusively escape across the source radius with the characteristic time for diffusive shock acceleration and found the corresponding, limiting Larmor radius which he had taken as a measure of the scattering mean free path. The maximum energy is then given as

$E_{\max }^{18}=\beta_{\mathrm{s}} \cdot Z \cdot B_{\mu G} \cdot L_{\mathrm{kpc}}$.

Here, $E_{\max }^{18}:=E_{\max } /\left(10^{18} \mathrm{eV}\right)$ is the maximum energy that can be achieved, $\beta_{\mathrm{s}}=V_{\mathrm{s}} / c$ where $V_{\mathrm{s}}$ is the shock velocity, $Z$ is the charge of the accelerated particle in units of the charge of the electron, $e$. Furthermore, $B_{\mu G}:=B /(1 \mu \mathrm{G})$ is the magnetic field of the acceleration region in units of $1 \mu \mathrm{G}$ and $L_{\mathrm{kpc}}:=$ $L /(1 \mathrm{kpc})$ is the size of the acceleration region in units of $1 \mathrm{kpc}$. Hillas (1984) also pointed out that Eq. (1) arose in a one-shot acceleration scheme due to the motion of a conductor in a magnetic field while the relationship of this condition to shock drift acceleration for an oblique shock is explored by Jokipii (1987). A second criterion of Hillas that the proton synchrotron loss time should not be less than the acceleration time, is easily met by proton shock acceleration if the shock is relativistic.

In an AGN environment the magnetic field can have values up to $B \sim 10^{-3} \mathrm{G}$ in the jet at a radius of $r \sim 1 \mathrm{kpc}$ and the field typically decreases inversely with the radius. This condition allows particle acceleration up to the highest energies required, i.e. $E_{\max }^{\mathrm{AGN}} \sim 10^{21} \mathrm{eV}$, see Biermann \& Strittmatter (1987). Radio galaxy hot spots allow acceleration of particles up to $10^{21} \mathrm{eV}$ and energies of $10^{19} \mathrm{eV}$ can be produced in radio galaxy lobes. Other suggested sources are not able to produce particles of sufficient energy because the field strengths available are insufficient to contain the particles.

GRBs may also accelerate protons to $5 \times 10^{20} \mathrm{eV}$. This is because fields up to $\Gamma B=10^{14} \mathrm{G}$ can be produced at an accretion torus located at about $6 \times 10^{6} \mathrm{~cm}$ radius in massive star collapse. Quantum mechanical dissipation limits the field amplification above this strength, see Lerche \& Schramm (1977), while the subsequent $1 / r$ field fall-off yields an $E_{\max }$ independent of $r$. Acceleration occurs without significant synchrotron losses, see Vietri (1995), Waxman (2000). The acceleration of particles during the prompt emission phase in highly-relativistic shocks ( $\Gamma=100-1000)$ is discussed in the following. Acceleration in external shocks during the slowing afterglow phase is also possible so that the spectra may resemble the AGN source spectra that we calculate and present in Sect. 3.

\section{The physical concept and the Monte Carlo simulations}

Begelman \& Kirk (1990) have claimed that most upstream field configurations at high shock boost factors $\Gamma$ appear superluminal. In superluminal shocks the particles are accelerated by the shock drift mechanism because there is no transformation into a de Hoffmann-Teller (HT) frame, see de Hoffmann \& Teller (1950), where $\boldsymbol{E}=\mathbf{0}$. As viewed in the shock rest frame, the particle is moving in a steep magnetic field gradient perpendicular to the shock surface. 
To transform from the normal shock frame (NSH) to the HT frame, we need to boost by a speed $V_{\mathrm{HT}}$ along the shock frame, where $V_{\mathrm{HT}}=V_{\mathrm{NSH}} \cdot \tan \psi$ ( $\psi$ is the angle between the magnetic field and the shock normal). Due to physical causality, this transformation is only possible if $V_{\mathrm{HT}}$ is less or equal to the speed of light. Thus, when $V_{\mathrm{NSH}}=c$, the limit is $\tan \psi=1$.

When $\tan \psi \leq 1$, the subluminal shock transformation case is applied. For all other cases, where a HT frame cannot be found due to a very high inclination in combination with a high shock velocity, the superluminal shock condition applies. While in the subluminal case particle transmission at the shock can be decided in the HT frame employing conservation of the first adiabatic invariant, in the superluminal case, computations are followed entirely in the fluid rest frames with reference to the shock frame, simply employed to check whether upstream or downstream shock conditions apply.

Meli \& Quenby (2003a,b) showed that a transformation from an initially isotropic rest frame distribution to an accelerated flow frame leads to a comoving frame field distribution lying close to the flow vector when the flow is highly relativistic. In general, flow into and out of the shock discontinuity is not along the shock normal, but a transformation is possible into the NSH frame to render the flows along the normal, see Begelman \& Kirk (1990). We assume that such a transformation has already been made.

Vietri et al. (2003) raise the question as to the correct reference frame in which jet acceleration should be viewed. For our calculations, we choose normalisation of our computed spectra to the total energy available to be performed in the shock frame. While the transformation affects the maximum energy obtained in the rest frame to some extent, the fact that escape from the beamed jet is mainly by motion perpendicular to the flow, means that we are chiefly involved in transforming a momentum vector perpendicular to the relative velocity of the reference frames, where there is no Lorentz correction. For X-ray production in shocks the relevant results are in the downstream frame.

The purpose of the Monte Carlo simulations is to find a solution to the particle transport equation for highly-relativistic flow velocities. The assumption of particle scattering by magnetic irregularities fixed in the plasma frame needs to be addressed. The Alfvén velocity, $V_{\mathrm{A}}$, for AGN jets with a maximum termination shock field of $3 \times 10^{-4} \mathrm{G}$, is deduced from an assumed equipartition with the electrons, responsible for the observed synchrotron radiation, see Laing (1981). An electron density of $10^{-2} \mathrm{~cm}^{-3}$ suggested by Faraday depolarisation measurement (Mc Carthy et al. 1987), and an assumed equal number of protons, yields $6 \times 10^{8} \mathrm{~cm} / \mathrm{s}$. However, modelling jet flow dynamics can suggest very low densities. For example, Laing \& Brindle (2001) find $10^{-30} \mathrm{gm} \mathrm{cm}^{-3}$, or an apparent Alfvén speed $>c$. For GRB jets a $100 \mathrm{G}$ field in the observer's frame is implied near the head of the blast after about 5 days, based on the argument in Sect. 1.1. A proton/electron number density of $10^{2} \mathrm{~cm}^{-3}$ between the forward and reverse shocks arises by modelling the jet interaction with a typical galactic medium Piran (1999). These numbers lead again to an apparent Alfvén speed in jets $>c$. This suggests that we use the relativistic theory expression for the incompressible Alfvénic mode, $V_{\mathrm{A}}=U_{\mathrm{A}} /\left(1+U_{\mathrm{A}}^{2} / c^{2}\right)^{1 / 2}$ where $U_{\mathrm{A}}=c B /(4 \pi W)^{1 / 2}$ and $W$ is the specific enthalpy, see Akhieser et al. (1975). In the high field limit, $V_{\mathrm{A}} \rightarrow c$ is in the field direction. Since we are considering relativistic plasma flow, it is necessary to take the plasma medium as a CR gas. Compressive hydrodynamic wave modes are possible with velocities up to $V_{\mathrm{cr}}=c / \sqrt{3}$ for an isotropic pressure tensor and, up to $V_{\mathrm{cr}} \approx c$ for a highly anisotropic distribution, see Webb (1987). The average second order fractional Fermi energy gain per collsion is $\left(V_{\mathrm{A}} / c\right)^{2}$. It is therefore possible that in each collision of mean free path along the field, the CR doubles its energy on average in a second order Fermi process. Moreover, numerical jet simulations suggest that large scale knot features travel at about $c / 5$ (van Putten 1997), so this energy gain may be overestimated, especially for the highest energy CRs. The time constant for this process can therefore be as low as $\tau_{F}=\lambda_{\text {down }} / c$ where $\lambda_{\text {down }}$ is the parallel mean free path downstream, where this acceleration is most effective. Furthermore, the standard diffusive shock acceleration time constant must be modified taking into account the relativistic speed up by a factor $\Gamma^{t}$, where $t$ varies between 2 and 0 as the number of shock crossings increases, as demonstrated by Meli \& Quenby (2003b) and by the subsequent computations in this paper. Since the upstream diffusion is the dominant factor here, the diffusive shock time constant for relativistic shock speeds is $\tau_{\mathrm{D}}=\lambda_{\text {up }} \cos ^{2} \psi / \Gamma^{t} c$, because it is the diffusion coefficient in the shock normal direction that counts. Hence, the ratio of acceleration times is $\tau_{\mathrm{F}} / \tau_{\mathrm{D}}=\Gamma^{t} \lambda_{\text {down }} / \lambda_{\text {up }} \cos ^{2} \psi$. If, as we argue below, $\lambda_{\text {up }}=\lambda_{\text {down }}$, diffusive shock acceleration is clearly more important. However, we cannot completely exclude scattering regimes where second order Fermi dominates and a different spectral form will emerge for the accelerated CRs. In the following, we neglect fluid frame acceleration beyond the region of trajectory intersection with the shock surface.

The scattering will be treated via a pitch angle scattering approach. In standard kinetic theory the spatial diffusion coefficients parallel and perpendicular to the field, $\kappa_{\|}$and $\kappa_{\perp}$, are related by $\kappa_{\perp}=\kappa_{\|} \cdot\left(1+\left(\lambda / r_{l}\right)^{2}\right)^{-1}$, Jokipii (1987). $\lambda$ is the parallel scattering mean free path and $r_{l}$ the Larmor gyroradius. In the well known Bohm Limit, $\lambda / r_{l}=1$, but interplanetary particle propagation studies referenced in Quenby \& Meli (2005) suggest $\lambda$ is several times the particle gyroradii $r_{l}$. Equation (9) of Quenby \& Meli (2005) is a simple, gyroresonance theory approximation to $\lambda$. Substitution of parameters corresponding to a turbulence field power of about one third of the mean field power, with the fluctuation spectrum existing over a spatial frequency range of about $2 \times 10^{4}$, yields $\lambda=10 r_{l}$. This value for $\lambda$ will be adopted in the following. Hence, in the shock normal, or in the $x$ direction, the diffusion coefficient is given by $\kappa=\kappa_{\|} \cos ^{2} \psi$, where $\kappa_{\|}=\lambda v / 3$ since we assume that $\kappa_{\|} \gg \kappa_{\perp}$. A guiding centre approximation is therefore used to follow propagation along field lines. Relativistic shocks generate strong small-scale turbulent magnetic fields downstream by the relativistic two stream instability, see Dieckmann et al. (2008), while the mean field strength also increases, according to simulations carried out by e.g. Medvedev (1999). In quasi-linear particle scattering theory, a particular particle "rigidity" (momentum to charge ratio) is resonantly scattered by waves at a cyclotron frequency which is of course proportional to field strength. For simplicity, we assume the ratios of downstream to upstream wave power and mean field strength are of similar magnitude, so $\lambda_{\text {down }}=\lambda_{\text {up }}$.

Gallant et al. (1999) have demonstrated analytically that particles entering the upstream region in a direction nearly normal to the shock can only experience small-angle pitch angle changes (pitch angle diffusion), $\delta \theta \leq 1 / \Gamma$, with $\delta \theta$ measured in the upstream fluid frame, for motion in a uniform field or a randomly oriented set of uniform field cells. The condition arises because particles attempting to penetrate upstream from the shock are swept back into the shock before they can scatter far in pitch angle. If we consider the ratio of initial energy to 
final energy, measured in the upstream or "primed" frame, after up to down to up transmission in an inclined shock, we have

$E_{f}^{\prime} / E_{i}^{\prime}=\Gamma^{2}\left(1+\beta_{r} \mu_{\rightarrow d}^{\prime}\right)\left(1-\beta_{r} \mu * \rightarrow u\right)$

where $\beta_{r}$ is the relative velocity of the frames as a fraction of $\mathrm{c}, \mu_{\rightarrow d}^{\prime}$ is the cosine of the particle velocity angle to the normal direction in the upstream frame at the moment of going downstream and $\mu * \rightarrow u$ is cosine of the particle velocity angle to the normal in the downstream frame at the moment of going upstream. For down to up crossing, $-\mu * \rightarrow u>B_{1}^{t} / B_{2}^{t}$ where $B_{1}^{t} / B_{2}^{t}$ is the inverse compression ratio. Up to down, the pitch angle scattering constraint is $\mu_{\rightarrow d}^{\prime} \approx-1+1 / \Gamma^{2}$. As pointed out by Baring (1999), $E_{f}^{\prime} / E_{i}^{\prime} \approx 2$. On the first up to down to up cycle with injection upstream and directed towards the shock, $E_{f}^{\prime} / E_{i} \approx \Gamma^{2}$, corresponding to the large angle scattering case of Quenby \& Lieu (1989). Since this constraint is largely dependent on the kinematic competition between upstream particle flow and the relativistic approach of the shock front, it is not critically dependent on the exact magnitude of the pitch angle scattering. Moreover, as shown by Quenby \& Meli (2005), blobs of high field scattering centres which could cause even large angle scattering, are allowed if the Larmor radius in the blob, $r_{l, b}$, satisfies $\left(\lambda / 2 \Gamma^{2}\right)>r_{l, b}$ where the parallel mean free path $\lambda=X r_{l, \text { ambient }}$ and $X \sim 10$. Such fields would allow substantial scattering before particles are swept back to the shock. This criterion, derived for parallel shocks, effectively applies in the oblique, subluminal case since in the upstream frame, the field direction is near the normal direction. To obtain a deflection $\sim 10 \Gamma^{-1}$, the constraint is relaxed to $b / B \gtrsim 2 \Gamma$ as the ratio of "blob" to ambient field, where $b$ is the perpendicular perturbation to the mean field, $B$. Hence, a relatively few field blobs, perhaps originating in strong instability in a hypernova collapse and with field strengths up to a factor 1000 stronger than the ambient, would be required to allow pitch angle scattering greater than $\delta \theta=1 / \Gamma$. For practical purposes, remembering it is the downstream scattering that is relevant to particle loss, it seems reasonable to use higher values of $\delta \theta$, within the pitch angle diffusion model.

A simple representation for the effect of the turbulence which relates to previous work, is to suppose the particle scatters $\delta \theta=N / \Gamma$ every $\lambda=10 r_{l}$. A transverse field perturbation changes the pitch angle in a quasi-linear theory, Kennel \& Petscheck (1966), by

$\delta \theta=\omega \frac{b}{B} \delta t$

in a time $\delta t$ due to a perpendicular perturbation, $b$, to the mean field, $B$, with cyclotron angular frequency, $\omega=e B / \gamma m_{\circ} c$. The particle moves in near gyroresonance with the wave in $b$. A pitch angle diffusion coefficient can then be derived

$D_{\theta}=\frac{\delta \theta^{2}}{\delta t}=\frac{\omega^{2}}{v_{\|}} \frac{P(k)}{B^{2}}$,

where $P(k)=P_{\circ} k^{s}$ is the power spectral density per unit wave number of $b^{2}$ at gyroresonance wave number $k=\omega / v_{\|}$. Note we have simplified the resonance condition to field structures which appear stationary to relativistic particles and correspond to wave propagation along the mean field. A particle then diffuses in pitch by a finite amount $\delta \theta$, during $\delta t$ given by

$\delta \theta^{2}=2 D_{\theta} \delta t$.

It is waves, of wave number $k$, fulfilling the gyroresonance condition, $k=\omega / v_{\|}$that cause the scattering of particles satisfying $\omega r_{l}=v_{\perp}$. We choose $\delta \theta$ to lie between $1 / \Gamma$ and $10 / \Gamma$, so on average a particle in an isotropic distribution, moving along the field at $c / \sqrt{3}$, scatters $5 / \Gamma$ after a time $10 \sqrt{3} r_{l} / c$ so that

$\delta \theta^{2}=\frac{25}{\Gamma^{2}}=\frac{2 \omega^{2}}{v_{\|} B^{2}} P_{\circ}(k) \frac{\omega^{s}}{v_{\|}^{s}} \frac{10 \sqrt{3} v_{\perp}}{c \omega}$.

To choose $\delta \theta$ independent of particle $\gamma$, that is of $\omega$, we require $s=-1$ for the spectral slope. Then we obtain a power spectrum relative to the mean field power

$\frac{P(k)}{B^{2}}=\frac{5}{4 \sqrt{2} \Gamma^{2}} k^{-1}$.

The total fractional power in the turbulence if resonating waves are present to scatter particles of between $\gamma=300$ and $\gamma=10^{12}$ is $1.4 / \Gamma^{2}$. Hence, the chosen pitch angle scattering model corresponds to a weak turbulence situation. Because quasi-linear theory for wave particle interactions is known to be an inexact approximation, the power spectrum we have presented must also be an approximation to the scattering model we employ. The choice of a fixed factor, 10, in the relation between $\lambda$ and $r_{l}$ acknowledges this inexactness. The spectrum is simply presented to provide a link with the work of others, especially Niemec \& Ostrowski (2005) who realise the field fluctuations employing a specified wave spectrum.

Our past studies of pitch angle scattering, see Meli \& Quenby $(2003 \mathrm{a}, \mathrm{b})$, where $\delta \theta \leq 1 / \Gamma$, suggested that the spectra became smoother as the magnetic field inclination angle to the shock normal decreased. Both a pronounced, plateau-like structure and increasing flattening developed towards the highest values of the shock boost factor $\Gamma$ studied. Additionally, at all inclination values used in the simulations, with $\Gamma$ limited to the range $10 \rightarrow 30$, the spectral form remained smooth. Ellison \& Double (2004), Stecker et al. (2007) and references therein have shown similar trends. In this more comprehensive study, the previous claims will be more thoroughly investigated.

For further details on the simulations, see Appendix A.

\section{Results}

\subsection{CR shock acceleration spectra}

The physical concepts and analytical approximations previously mentioned are employed as the basis for Monte Carlo trajectory simulations for relativistic superluminal and subluminal shocks. Computed particle spectra will be presented with special focus on the relation between the boost factor, $\Gamma$, for a given astrophysical source and the resulting spectral features. Of particular relevance is Sect. 3.2 which is devoted to the contribution of these sources to the observed diffuse CR spectrum.

\subsubsection{Superluminal shock spectra}

Initially, we present simulations for relativistic superluminal shocks as described in the previous section and the Appendix. Unlike our previous work which was limited to a large angle scattering description, pitch angle scatter is now employed. The guiding centre approximation is used except for motion within one complete particle helical cycle from the shock. Since a transformation into the HT frame as described in Sect. 2 is not possible, the particles are followed by trajectory integration in the appropriate fluid rest and NSH frames. This, in practise, simulates the shock drift mechanism. We follow the helical trajectory 


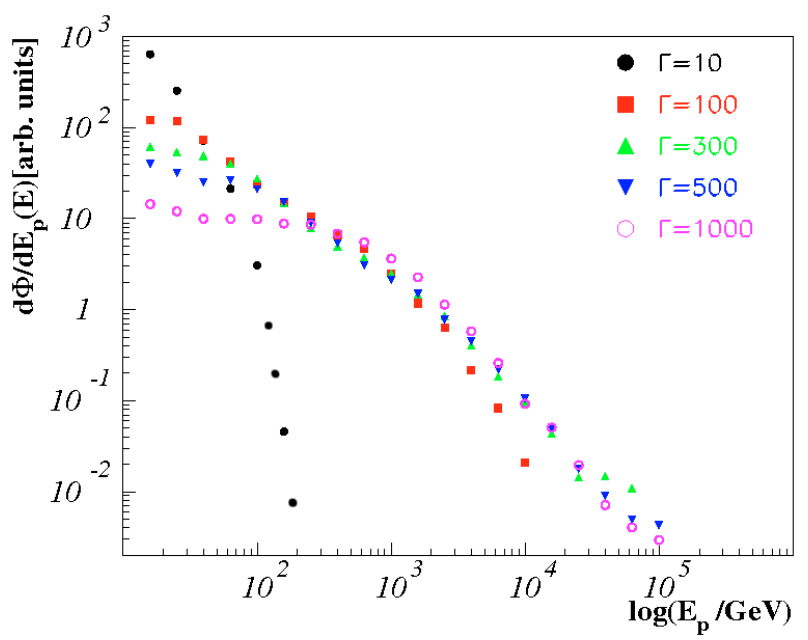

Fig. 1. Superluminal, relativistic spectra at $\psi=76^{\circ}$. Boost factors are varied between $\Gamma=10,100,300,500,1000$. Spectra for different inclination angles $\psi$ are comparable.

of the particle until it intersects the shock front, applying pitch angle scatter $[1 / \Gamma \leq \delta \theta \leq 10 / \Gamma, \phi \in(0,2 \pi)]$ right up to the shock interface. Performing a variety of simulation runs demonstrated that the results were almost independent of $\psi$, the angle between the magnetic field and the shock normal. We therefore illustrate the results with a simulation run with a typically large inclination angle, $\psi=76^{\circ}$, employing a range of boost factors. The resulting spectra are presented in the shock frame on the downstream side to ensure comparability with following calculations.

The resulting particle spectra for $\Gamma=10,100,300,500$ and 1000 are displayed in Fig. 1. These plots indicate that for mildly-relativistic shocks $(\Gamma=10)$, the mechanism is efficient at acceleration particles up to energies $E_{\mathrm{p}}<10^{2} \mathrm{GeV}$. More highly-relativistic shocks $(\Gamma \geq 100)$ can produce particles up to $E_{\mathrm{p}}<10^{5} \mathrm{GeV}$. The upper limit of significant acceleration is $\sim \Gamma^{2}$, corresponding to only one complete particle crossing cycle with the majority of particles either failing to return a second time or only returning at angles close to the normal. In the region of efficient acceleration, the spectra approximately follow power-laws with spectral indices lying between $\sim 2.0-2.3$. In contrast, for the case of large angle scattering, previously studied by Meli \& Quenby (2003b), the spectra could not be described by power-laws, but exhibited a convex shape terminating in a steep energy cut-off.

We conclude that superluminal, relativistic shocks are not efficient accelerators for very high energy particles and are unlikely to contribute to observable effects, discussed in more detail in Sect. 3.2. These conclusions concur with the work of Niemec \& Ostrowski (2007). On the other hand, while superluminal shocks cannot contribute to the observed spectrum of charged UHECRs, a contribution to the neutrino- and $\mathrm{TeV}$-photon background arising from proton-photon or protonproton interactions, is still possible as discussed in Sect. 4.

\subsubsection{Subluminal shock spectra}

Particle spectra produced in relativistic subluminal shocks have been calculated for three different inclination angles in the shock frame, $\psi=23^{\circ}, 33^{\circ}$ and $43^{\circ}$ which we chose as representing the possible range of subluminal shock angles likely in astrophysical sources. In Appendix B, simulated particle spectra are presented for the entire energy range of particle energy considered,
$E_{\mathrm{p}}=10^{2}-10^{12} \mathrm{GeV}$, for a range of boost factors, $\Gamma=10$ to $\Gamma=1000$ and for all three angles. An important result lies in the trend of spectral smoothness. They appear as smooth power-laws when the shocks are mildly-relativistic, $\Gamma \leq 30$. As the boost factor increases, bumps appear in the spectra $(\Gamma>100)$. The plateau-like parts of the spectra at higher energies and higher boost factors are caused by particles continuing to undergo significant acceleration in a second cycle. The lower energy part of the spectrum is dominated by particles undergoing one acceleration shock crossing while the second bump in the spectrum represents particles experiencing two acceleration shock crossings. In this first complete shock cycle crossing from upstream to downstream to upstream, the energy gain is a factor $\Gamma^{2}$, operating on an injection energy already $\approx \Gamma$ in magnitude. Subsequent crossings become smoother since the energy gain is expected to be limited to $\sim 2$ and there is a statistical smoothing of the energy gains. At the highest boost factors we investigate, this smoothing regime is not reached.

In order to get a representative picture, we employ particle spectra averaged over the three inclination angles at a particular $\Gamma$, in all following calculations. This should give a more realistic estimate of the diffuse particle flux from extragalactic sources, since a range of angles is likely to occur in the AGN and GRB shocks. We concentrate on the highest energies because observation of particle-induced air showers indicate that at energies between $10^{9.5} \mathrm{GeV}$ and $10^{10.5} \mathrm{GeV}$ the origin of the charged CR flux is extragalactic and that this flux is distinct from a dominant, galaxy produced component at lower energies. A power-law fit is made to the simulated spectra between $10^{9.5} \mathrm{GeV}$ and $10^{10.5} \mathrm{GeV}$. Figure 2 shows the averaged, simulated spectra between $10^{8.5} \mathrm{GeV}$ and $10^{11} \mathrm{GeV}$. At even higher energies, the spectra will be modified in practise by the absorption of protons due to interactions with the cosmic microwave background. While the normalisation of the spectra is arbitrary since it is dependent on the number of injected particles in the Monte-Carlo simulation, the spectral index can be compared to the observed CR index in the same energy range. Table 1 shows the variation of the spectral index with the boost factor. While mildly-relativistic shocks show indices around $\alpha_{\mathrm{p}} \approx 2$, highlyrelativistic shocks with $\Gamma>100$ have flatter spectra with indices between $0.7<\alpha_{\mathrm{p}}<1.5$. Particle spectra emitted during the prompt phase of GRBs $(\Gamma>100)$ will therefore appear much flatter than AGN particle spectra $(\Gamma \sim 10)$. This has important implications for the interpretation of the origin of the UHECR spectrum, as will be discussed in a following subsection.

Stecker et al. (2007) investigating parallel shocks up to $\Gamma=$ 30 found an increase in structure in the spectral shape and a decrease in slope as $\Gamma$ increased with a dependence $E^{-1.26}$ at $\Gamma=30$. These trends are shown in this work, extending to far higher $\Gamma$ factors and for a more general set of subluminal shock inclination angles. Bednarz \& Ostrowski (1998) employed pitch angle scattering and varying cross-field diffusion coefficients and found that at low $\Gamma$, steep spectra occurred at large inclination angles but all values of these parameters seemed to produce spectral slopes of -2.2 at $\Gamma=243$. In contrast Meli \& Quenby (2003a) found spectra flatter than $E^{-2}$ for parallel shocks as $\Gamma \rightarrow$ 1000. In addition, related work by Niemec \& Ostrowski (2005) with wave spectra $P(k) \sim k^{-1 \rightarrow 1.5}$, found spectra flatter than $E^{-2}$ with noticeable spectral structure in inclined, subluminal shocks at upstream velocities of $0.5 c$ and a weakly perturbed field. Their trajectory integrations took into account cross field diffusion. Baring (2004) however, cites previous work with no cross field diffusion which found that significant acceleration 


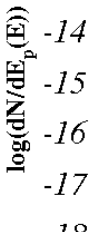
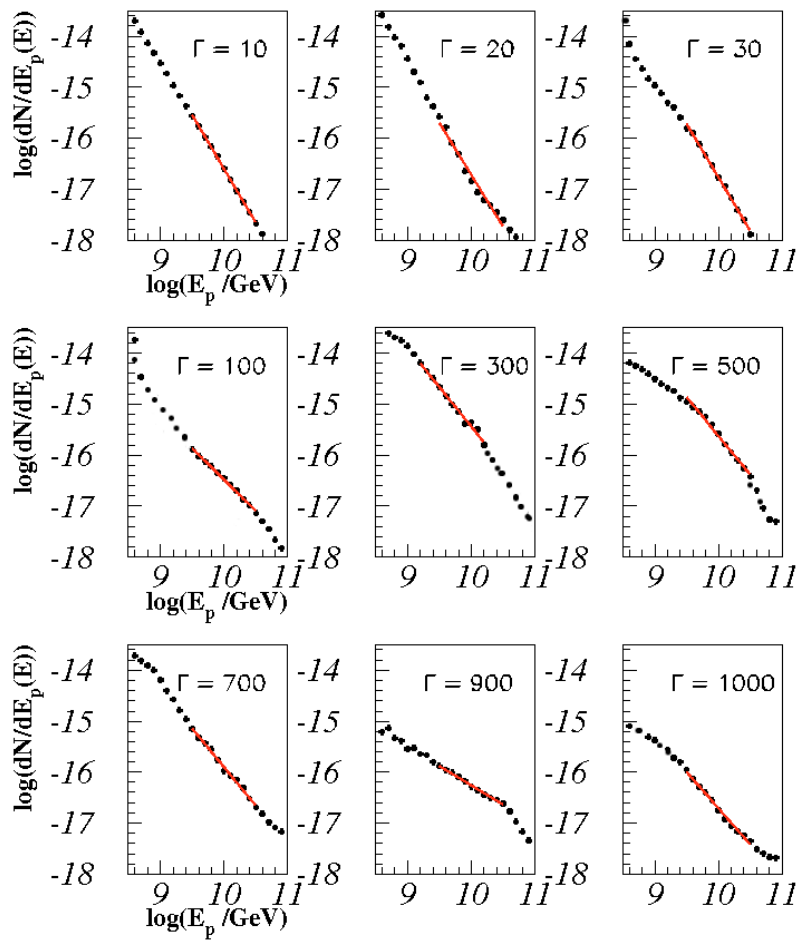

Fig. 2. Subluminal spectra averaged over the three angles for different $\Gamma: \Gamma=10,20,30$ is displayed in the first row, in the middle, $\Gamma=100,300,500$ is shown and $\Gamma=700,900,1000$ is the bottom row. The black circles in each graph represent the simulation result. The red lines show the single power-law for comparison.

Table 1. Spectral indices for a single power-law comparison for subluminal shocks. The spectral fits were made between $10^{9.5} \mathrm{GeV}$ and $10^{10.5} \mathrm{GeV}$ in order to be comparable to the observed CR flux at the same energies. The uncertainty from the fit is less than $10 \%$ if we assume an accuracy of the simulation is better than $\Delta \log \left(\mathrm{d} N / \mathrm{d} E_{\mathrm{p}}\right) \sim 0.5$, which is a conservative estimate.

\begin{tabular}{l|lllllllll}
\hline \hline$\Gamma$ & 10 & 20 & 30 & 100 & 300 & 500 & 700 & 900 & 1000 \\
\hline$\alpha_{\mathrm{p}}$ & 2.1 & 2.0 & 2.1 & 1.2 & 1.5 & 1.5 & 1.5 & 0.7 & 1.4 \\
\hline
\end{tabular}

in relativistic, inclined shocks was limited to inclination angles $\leq 25^{\circ}$. It is important to note here that it is not clear whether the very steep spectra quoted for higher inclination angles are due to the steep slope at the edge of the first plateau we mention in this work (see Appendix B), or whether there is a significant difference from our modelling. A high perpendicular diffusion coefficient, high inclination shock situation might be expected to be equivalent to a high scattering, parallel shock regime, but this possibility does not seem to reconcile the various conflicting results just discussed. There is an agreement that at very high inclinations, significant acceleration above that due to a single shock cycle is ruled out. Also, there seems to be a developing consensus that high $\Gamma$, subluminal shocks result in flatter spectra than $E^{-2}$. Dingus (1995) provides gamma-ray burst evidence for relativistic electron spectra with relatively flat slopes with negative exponents at least as low as $\approx 2$.

Results indicating a variety of possible slopes are also consistent with radio data on the electron spectra injected at terminal hotspots in the lobes of powerful FR-II radio galaxies where no single, universal power-law is found, as shown by Rudnick et al. (1994) and Machalski et al. (2007) among others.

\subsection{Diffuse $C R$ spectra from GRBs and AGN}

The source spectra derived previously can be translated into an expected diffuse proton flux from astrophysical sources by folding the spectra with the spatial distribution of the sources. In this section, AGN and GRBs are used as potential candidates because these are the sources with the highest observed output in relativistic electrons. Since the particle spectra are strongly dependent on $\Gamma$, it is important to discuss which spectra to use for these two source classes. Spectral choice is investigated in the next subsection before the actual calculation of the diffuse spectra is shown. In the last subsection, the results of our calculations are compared to $\mathrm{CR}$ data.

\subsubsection{AGN and GRBs - intrinsic spectra}

Spectra derived from the predictions of the AGN and GRB acceleration models, according to their appropriate boost factors, will be fitted to the CR energy range $10^{9.5} \mathrm{GeV}$ to $10^{10.5} \mathrm{GeV}$ which is almost certainly of extra-galactic origin.

The boost factor deduced from electron synchrotron observation can vary significantly in the case of GRBs. While the majority of sources are estimated to have boost factors around $\Gamma \approx 300$, more moderate values down to $\Gamma=100$ or more extreme values up to $\Gamma=1000$ are believed to occur. However, the exact distribution of GRBs $\Gamma$ factors cannot be determined. In many cases, only upper limits can be given. In addition, there may be hidden bursts not observed with GRB satellite experiments. It is therefore not useful to model a detailed distribution of boost factors for GRBs, while the simulation results connecting the spectral index of the spectrum with a boost factor are subject to uncertainty, as is implied by the absence of a monotonic trend in Table 1.

It was shown that for boost factors of $\Gamma>100$, the source spectra lie between $E_{\mathrm{p}}{ }^{-1.5}$ and $E_{\mathrm{p}}{ }^{-0.7}$. A conservative estimate for the flattened GRB spectra will be adopted using

$\frac{\mathrm{d} \Phi_{\mathrm{GRB}}}{\mathrm{d} E_{\mathrm{p}}} \propto E_{\mathrm{p}}^{-1.5}$

The situation is simpler for AGN, as the maximum value of the shock's boost factor is limited to between $\Gamma=10$ and $\Gamma=30$. Here, all computed spectra cluster around a value of $E_{\mathrm{p}}{ }^{-2.1}$. Therefore, the AGN spectrum will be taken as

$\frac{\mathrm{d} \Phi_{\mathrm{AGN}}}{\mathrm{d} E_{\mathrm{p}}} \propto E_{\mathrm{p}}^{-2.1}$

\subsubsection{From CR shock acceleration spectra to a diffuse spectrum}

The diffuse spectrum as measured at Earth depends on several factors:

- Single source spectra at the source $\mathrm{d} \Phi / \mathrm{d} E_{\mathrm{p}}$. The spectral behaviour was already discussed in the previous subsection. To account for particle propagation, adiabatic energy losses need to be considered as $E_{\mathrm{p}}(z)=E_{\mathrm{p}} \cdot(1+z)$. Here, $E_{\mathrm{p}}(z)$ is the energy as observed at a source at redshift $z$ and $E_{\mathrm{p}}$ is the corresponding energy observed at Earth. Diffusive propagation in the magnetic field between clusters is assumed to involve only small angle scattering with preservation of spectral shape. Anisotropy in the source distribution is neglected. In addition, we consider pure proton spectra so that spallation effects are not present. 
- Source evolution $g(z)$ : it is assumed that both AGN and GRBs follow the SFR to determine the number density evolution with comoving volume, see for example, Hasinger et al. (2005) in the case of AGN and Pugliese et al. (2000) in the case of GRBs. A large sample of radio quiet AGN selected at X-ray wavelengths was investigated by Hasinger et al. (2005). The comoving density $\mathrm{d} n / \mathrm{d} V(z)$ is given as

$\frac{\mathrm{d} n}{\mathrm{~d} V}(z) \propto \begin{cases}(1+z)^{m} & \text { for } z<z_{1} \\ \left(1+z_{1}\right)^{m} & \text { for } z_{1}<z<z_{2} \\ \left(1+z_{1}\right)^{m} \cdot 10^{k \cdot\left(z-z_{2}\right)} & \text { for } z>z_{2},\end{cases}$

with the parameters $m=5.0, z_{1}=1.7, z_{2}=2.7$ and $k=$ -0.43 . The total redshift evolution $g(z)$ further includes multiplying the comoving volume $\mathrm{d} V / \mathrm{d} z$ with a factor $1 /\left(4 \pi d_{L}^{2}\right)$ to account for the decrease of the flux $L$ with the luminosity distance $d_{L}$, neglecting a possible travel limitation to the distance reached by significant magnetic scattering. Therefore,

$g(z)=\frac{\mathrm{d} n}{\mathrm{~d} V}(z) \cdot \frac{\mathrm{d} V}{\mathrm{~d} z} \cdot\left(4 \pi d_{L}^{2}\right)^{-1}$.

For simplicity, this model is used for both AGN and GRBs. Although deviations between the SFR scenarios of AGN and GRBs are expected, the approximation that both follow the distribution of radio quiet X-ray AGN is reasonable, Hasinger et al. (2005): the deviations being expected to be negligible with respect to general uncertainties arising from assumptions about the acceleration region.

- Absorption of protons at the highest energies: protons at $E_{\mathrm{p}}>5 \times 10^{19} \mathrm{eV}$ are absorbed due to interactions with the cosmic microwave background as was recently confirmed by the Auger experiment, see Yamamoto et al. (2007).

Therefore, the diffuse spectrum resulting from the propagation of a single source spectra is modified by a further factor $\exp \left[-E_{\mathrm{p}}(z) / E_{\text {cut }}(z)\right]$. Here, $E_{\text {cut }}(z)=5 \times 10^{19} \cdot(1+z)^{2} \mathrm{eV}$ is the cut energy, assuming that the mean free path of the protons is much shorter than the distance between Earth and source ${ }^{1}$.

- The normalisation of the diffuse spectrum: because the calculated particle spectra are given in arbitrary units, normalisation of the overall spectrum as measured at Earth is achieved using observation.

- In the case of superluminal sources, normalisation of the expected signal follows from the most restrictive upper limit on the neutrino signal from extraterrestrial sources given by the AMANDA experiment, see Achterberg et al. (2007)

$$
E_{v}^{2} \frac{\mathrm{d} N_{v}}{\mathrm{~d} E_{v}}<7.4 \times 10^{-8} \frac{\mathrm{GeV}}{\mathrm{s} \mathrm{sr} \mathrm{cm^{2 }}}
$$

With an average $E^{-2}$ spectrum for both neutrinos and protons, the spectra are connected by assuming that the expected neutrino energy fluence is a fraction $q$ of the proton spectrum

$$
\int \frac{\mathrm{d} N_{v}}{\mathrm{~d} E_{v}} E_{v} \mathrm{~d} E_{v}=q \cdot \int \frac{\mathrm{d} N_{\mathrm{p}}}{\mathrm{d} E_{\mathrm{p}}} E_{\mathrm{p}} \mathrm{d} E_{\mathrm{p}},
$$

with $q=1 / 40$, since only $20 \%$ of the proton flux goes into pion production via the delta resonance, $1 / 2$ of the remaining flux goes into the charged pion component of which 1/4 goes into neutrinos, see e.g. Becker (2008).

\footnotetext{
${ }^{1}$ At a redshift of $z=0$, the interaction of protons with the CMB happens at $E_{\text {cut }}(z=0) \cdot E_{\gamma}(z=0)=\left(m_{\Delta}^{2}-m_{\mathrm{p}}^{2}\right) / 4$. At a redshift $z$ and considering the redshift of the $\mathrm{CMB}$, this equation becomes $E_{\text {cut }}(z)=5 \times$ $10^{19} \cdot(1+z)^{2} \mathrm{eV}$.
}

- In the case of subluminal sources, using neutrino flux limits leads to an excess above the observed spectrum of charged CRs, since the limits are not stringent enough yet. Instead, the measured CR spectrum above the "ankle" is used to estimate the contribution from subluminal sources. The CR energy flux above the ankle is given by Waxman \& Bahcall (1997), Waxman \& Bahcall (1999) $j_{E}\left(E_{\min }=3 \times 10^{18} \mathrm{eV}\right):=$

$$
\int_{3 \times 10^{18} \mathrm{eV}} \frac{\mathrm{d} N_{\mathrm{p}}}{\mathrm{d} E_{\mathrm{p}}} E_{\mathrm{p}} \mathrm{d} E_{\mathrm{p}} \approx 10^{-7} \mathrm{GeV} \mathrm{cm}^{-2} \mathrm{~s}^{-1} \mathrm{sr}^{-1} \text {. }
$$

It is expected that this contribution comes from a combined signal from AGN and GRBs. In the following it is assumed that the fraction of UHECRs coming from AGN, contributes a fraction $0<x<1$. Therefore, the fraction of UHECRs from GRBs is $(1-x)$.

Thus, the total spectrum as observed at Earth is given as

$$
\begin{aligned}
\frac{\mathrm{d} N_{\mathrm{p}}}{\mathrm{d} E_{\mathrm{p}}}= & A_{\mathrm{p}} \int_{z_{\min }}^{z_{\max }}\left(x \cdot \frac{\mathrm{d} \Phi_{\mathrm{AGN}}}{\mathrm{d} E_{\mathrm{p}}}\left(E_{\mathrm{p}}(z)\right)+(1-x) \cdot \frac{\mathrm{d} \Phi_{\mathrm{GRB}}}{\mathrm{d} E_{\mathrm{p}}}\left(E_{\mathrm{p}}(z)\right)\right) \\
& \times(1+z)^{-1} \cdot \exp \left(-\frac{E_{\mathrm{p}}(z)}{E_{\text {cut }}(z)}\right) \cdot g(z) \mathrm{d} z
\end{aligned}
$$

The minimum redshift is set to $z=0.001$. This excludes only the closest AGNs which contribute less than $1 \%$ according to newest results of The Pierre Auger Collaboration (2007), Becker \& Biermann (2008). The maximum redshift is taken to be $z_{\max }=7$. As the main contribution comes from redshifts of $z \sim 1-2$ due to the high number of sources at these redshifts, the exact values of the integration limits are not crucial.

\subsubsection{Comparison with the observed cosmic ray spectrum}

The diffuse spectrum measured at the Earth is shown in Fig. 3. Data points from a selection of experiments are shown. Our calculated spectra from superluminal and subluminal shocks are displayed as the dashed and solid lines.

It appears from Fig. 3, that for superluminal shocks, the only possible contribution to the measured CR spectrum is around the knee. It is expected, however, that the effective flux is actually even lower, because the normalisation is based upon the assumption that the contribution cannot be more than the current neutrino flux limits permit. Therefore, the calculated flux can be considered as an absolute upper limit. Here, the fraction of AGN protons has been chosen to be $x=0.5$, assuming that $50 \%$ of the signal is produced by AGN and $50 \%$ by GRBs.

The subluminal shock case has been investigated for different scenarios. The upper line represents a spectrum that would be produced by AGN only $(x=1)$. The lower line represents a pure GRB spectrum $(x=0)$. The flux is too low to explain the observed component right above the ankle and too high towards higher energies if a significant contribution comes from GRBs. This is due to the flatness of the GRB spectra, and it seems that these flat spectra do not fit the present observations. Thus, AGN are the favoured sources for the production of UHECRs. Figure 4 shows the CR spectrum at the highest energies, multiplied by $E^{2.7}$ in order to have a clearer view of the features in the spectrum. The upper and lower solid lines represent the same predictions for shocks as in Fig. 3. The middle line assumes that $50 \%$ is made up by AGN and the remaining 50\% comes from GRBs. The normalisation fits the HiRes data. There is a discrepancy between the normalisation of HiRes and Auger data, which is not entirely understood yet, but is probably due to systematic errors in the energy and flux determination of the experiments. 


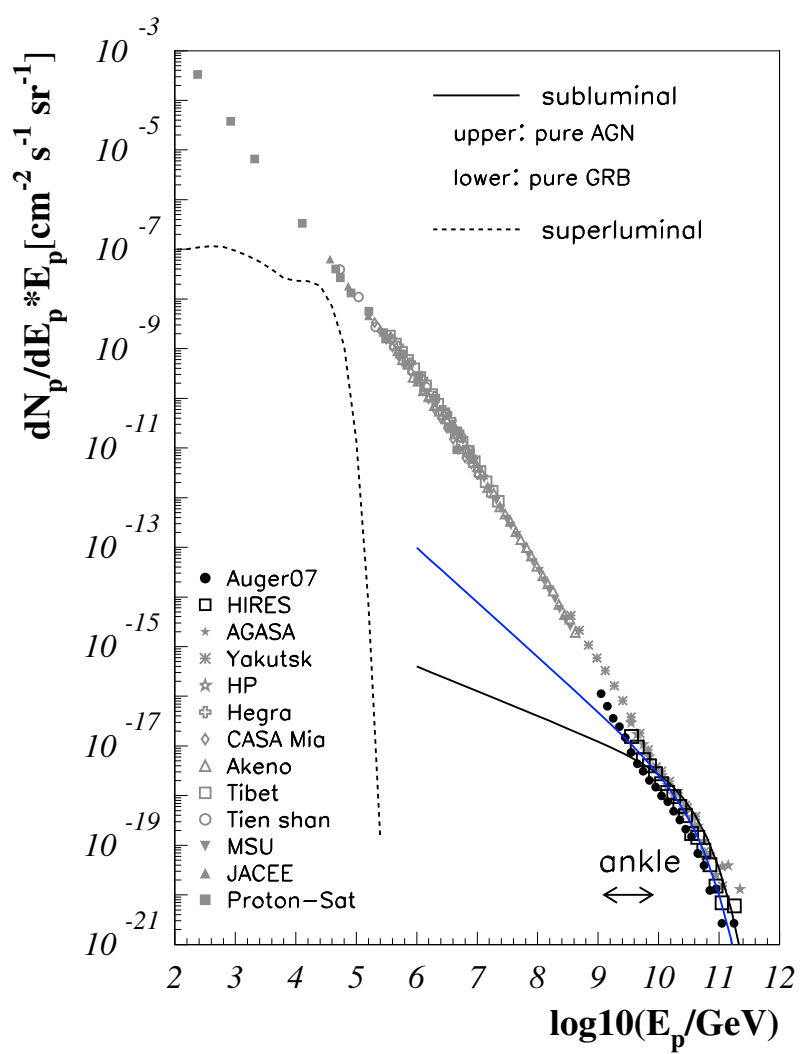

Fig. 3. The maximum predicted diffuse flux from GRBs and AGN with superluminal shock fronts (dashed line) and subluminal shocks (solid blue and black lines). For subluminal sources, the upper line is a pure AGN-produced spectrum, the lower line represents a pure GRB spectrum. The flux is compared to the measured CR spectrum. Data points are taken from the different experiments: Auger - Yamamoto et al. (2007); HiRes The High Resolution Fly's Eye Collaboration (2002); AGASA Yoshida et al. (1995); Yakutsk - Krasilnikov et al. (1985); Haverah Park - Ave et al. (2001); HEGRA - Aharonian et al. (1999); CASAMIA - Glasmacher et al. (1999); Akeno - Krasilnikov et al. (1985); Tibet - Ozawa et al. (2003); Tien Shan - Antonov et al. (1995); MSU Khristiansen et al. (1994); JACEE - Asakimori et al. (1995); ProtonSat - Grigorov et al. (1975); KASCADE - Antoni et al. (2005). In the case of superluminal sources, $50 \%$ is assumed to come each from GRB and $50 \%$ from AGN.

Therefore, the Auger data are renormalised at $10^{10.3} \mathrm{GeV}$ to match the normalisation of the HiRes data. With an assumed significant contribution from GRBs, it seems difficult to explain the observed spectrum. Within the uncertainties of the experimental data, our predicted spectra fit very well the measured spectra from $\sim 10^{9.7} \mathrm{GeV}$ if pure AGN spectra are assumed. The flux is slightly too low for the first data points above the ankle, between $10^{9.5} \mathrm{GeV}$ and $10^{9.7} \mathrm{GeV}$. The steepening of the spectrum at lower energies cannot be due to some contribution from sources with a high field inclination to the normal because we have shown that such superluminal shocks cannot produce energies above about $10^{10.5} \mathrm{GeV}$. It is possible that the steepening arises from the addition of subluminal sources with varying maximum energy, determined by varying maximum field strengths, so only a fraction of the sources reach the highest energies of $10^{21} \mathrm{eV}$. Ahlers et al. (2005), fitting HiRes data to a cosmological source distribution similar to ours, find some steepening around $10^{9} \mathrm{GeV}$ due to details of the photo-production propagation function. While these effects suffice to explain the small difference at the lowest energies above the ankle with respect to

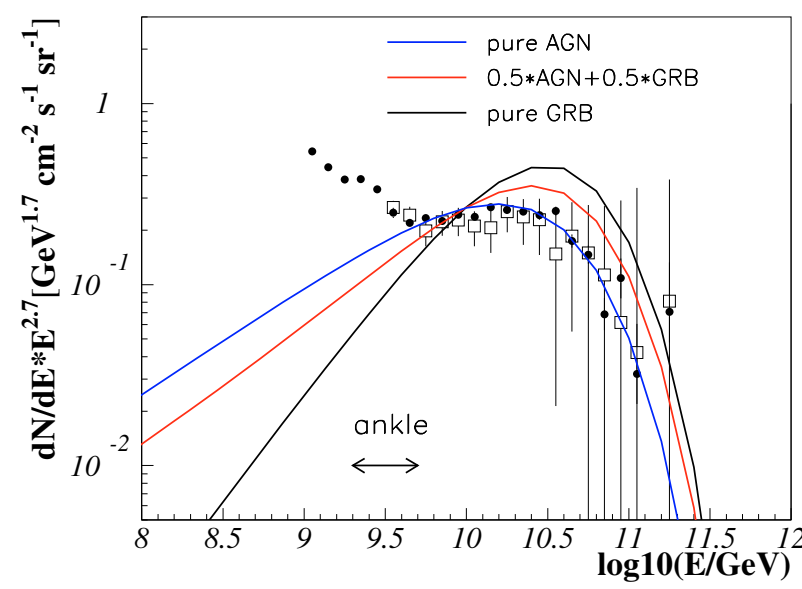

Fig. 4. Spectrum of UHECRs multiplied by $E^{2.7}$. Data points from Auger, Yamamoto et al. (2007) and HiRes, The High Resolution Fly's Eye Collaboration (2002). The solid lines represent the same predictions as presented in Fig. 3. Auger data have been renormalised at $10^{10.3} \mathrm{GeV}$ to HiRes data and the calculated spectra have also been normalised to HiRes data. The data can be described well by a pure AGN spectrum (blue line) within experimental uncertainties and test particle acceleration accuracy. The red line is a mixture of 50\% GRBs contribution and 50\% AGN contents, the black line is a pure GRB spectrum.

the AGN based spectra, they cannot explain the large differences between the data and the GRB spectral predictions.

\section{Implications for high energy neutrino and photon astronomy}

Figure 3 has an interesting implication for neutrino and $\mathrm{TeV}$-photon astronomy. Neutrinos and $\mathrm{TeV}$ photons are produced in proton-photon or proton-nucleon interactions, see e.g. Becker (2008) for a review,

$p \gamma \longrightarrow \Delta^{+} \longrightarrow \begin{cases}p \pi^{0}, & \text { fraction } 2 / 3 \\ n \pi^{+}, & \text {fraction } 1 / 3\end{cases}$

$p p \longrightarrow \pi^{+} \pi^{-} \pi^{0}$.

The decay of the $\pi^{0}$ leads to high energy photon emission, and $\pi^{ \pm}$particles produce neutrinos. The photon signal at $\mathrm{TeV}$ energies is not unique, since leptonic processes like Inverse Compton scattering contribute at the same energies. Therefore, the best, unambiguous way of identifying the hadronic interactions are neutrino observations.

Models for neutrino emission in AGN are presented in e.g. Mannheim (1995), Mannheim et al. (2001), Stecker (2005), Becker et al. (2005), where it is assumed that protons accelerated in AGN jets can interact with different photon fields to produce neutrinos.

Proton-photon interactions in the prompt phase of GRBs can lead to neutrino and $\mathrm{TeV}$-photon production. In the first approach by Waxman \& Bahcall (1997), Waxman \& Bahcall (1999), it is assumed that GRBs are the sources of UHECRs to calculate the neutrino spectrum. According to our results in the previous sections, GRBs are unlikely to be the sources of UHECRs and the model does not hold anymore. However, further developments of the model normalise the flux to the electromagnetic output rather than to the flux of UHECRs, see e.g. Guetta et al. (2004), Becker et al. (2006), Murase \& Nagataki (2006). Here, the protons do not need to be accelerated to the highest energies: the photon field of the prompt emission of GRBs has 
characteristic energies of around $\sim 100 \mathrm{keV}-1 \mathrm{MeV}$, and can reach energies up to $>100 \mathrm{MeV}$, see e.g. Schneid et al. (1992), González et al. (2003), Hurley et al. (1994). The proton energy necessary to produce a Delta resonance, and with these $\mathrm{TeV}$-photons and neutrinos, is given as

$E_{\mathrm{p}} \geq \frac{\Gamma^{2}}{(1+z)^{2}} \frac{m_{\Delta}^{2}-m_{p}^{2}}{4} \cdot E_{\gamma}^{-1}$.

Here, $m_{\Delta}$ is the mass of the Delta resonance, $m_{\mathrm{p}}$ is the proton's mass, $z$ is the redshift of the source and $E_{\gamma}$ is the characteristic photon energy. The proton energy required for the process is therefore given as

$E_{\mathrm{p}} \geq 10^{7} \cdot(1+z)^{-2} \cdot\left(\frac{\Gamma}{300}\right)^{2} \cdot\left(\frac{E_{\gamma}}{1 \mathrm{MeV}}\right)^{-1} \mathrm{GeV}$.

Thus, with redshifts typically of the order of $z=1$ and boost factors of around $\Gamma=300$, the proton energy sufficient for neutrino and $\mathrm{TeV}$-photon production is as low as

$E_{\mathrm{p}} \sim 10^{6} \mathrm{GeV}$.

This opens the possibility of neutrino production from sources which are not observable in charged UHECRs. While the lower energy spectra of extragalactic, charged CRs cannot be observed due to the high galactic background, neutrinos may serve to investigate those sources further.

Using the same reasoning as above, we can conclude that charged UHECRs from superluminal spectra may not be observed in charged CRs, but are good candidates for the production of high energy neutrinos and photons from AGN or GRBs. Those shocks may produce low energy spectra of much higher intensity than subluminal shocks, which are simply hidden due to the galactic background. Neutrinos and photons, on the other hand, point back to the original source, and may be identified. We can use the maximum energy of the proton spectra to calculate the energy of the photon spectra which are necessary to produce high energy photons and neutrinos by using Eq. (18),

$$
\begin{aligned}
E_{\gamma} & \geq \frac{\Gamma^{2}}{(1+z)^{2}} \frac{m_{\Delta}^{2}-m_{\mathrm{p}}^{2}}{4} \cdot E_{p}^{-1} \\
& =10^{7} \cdot(1+z)^{-2} \cdot\left(\frac{\Gamma}{300}\right)^{2} \cdot\left(\frac{E_{\mathrm{p}}}{1 \mathrm{GeV}}\right)^{-1} \mathrm{MeV} .
\end{aligned}
$$

For mildly relativistic superluminal shocks of $\Gamma \sim 10$ occuring in $\mathrm{AGN}$, superluminal proton spectra reach up to $E_{\mathrm{p}} \sim$ $100 \mathrm{GeV}$. This requires photon energies of $E_{\gamma} \geq 100 \mathrm{MeV}$. These high energies can be produced by Inverse Compton scattering of synchrotron or external photons with the accelerated electrons. A catalogue of AGN with photon emission above $100 \mathrm{MeV}$ has already been presented by the EGRET experiment, Sreekumar et al. (1998), and more sources are likely to be identified when GLAST is launched this summer, Gehrels \& Michelson (1999).

Highly relativistic superluminal shocks with $\Gamma>100$ may accelerate protons to energies of $10^{5} \mathrm{GeV}$. This requires photon energies of $E_{\gamma} \geq 10 \mathrm{MeV}$. This is about one order of magnitude above the characteristic energy, but the photon spectrum is likely to extend to energies above $100 \mathrm{MeV}$ as already observed for more than 30 GRBs, see e.g. Schneid et al. (1992), González et al. (2003), Hurley et al. (1994). If mildly relativistic internal GRB shocks are to contribute to neutrino production, photon energies in excess of $1 \mathrm{GeV}$ are required. Such sources would also need to be insignificant producers of UHECRs, to satisfy the discussion of Sect. 3.2.2.

In conclusion, low energy proton spectra from extragalactic sources cannot be observed directly due to the high galactic background of CRs, but they may be detected indirectly by means of high energy neutrino and photon spectra.

\section{Summary and conclusions}

In this work we have presented Monte Carlo simulation studies of the acceleration of test particles in relativistic, subluminal and superluminal shock environments. The source candidates discussed were AGN jets with mildly-relativistic shocks of boost factors of $\Gamma \approx 10-30$ and GRB outflows with highly-relativistic shocks, $100<\Gamma<1000$. The resulting particle spectra were used to calculate a contribution to the diffuse CR spectrum.

Particle spectra have been obtained with varying shock boost factor $\Gamma$ and shock obliquity, i.e. the inclination angle between the shock normal and the magnetic field, $\psi$. Only subluminal shocks are able to accelerate significant numbers of particles up to $10^{12} \mathrm{GeV}$, while superluminal shocks are only effective up to $\sim 10^{5} \mathrm{GeV}$ for the $\Gamma$ values chosen. Flat spectra are found for very high subluminal shock boost factors, but for superluminal shocks the spectral indices stay roughly constant between values of 2.0 to 2.3 in the limited region of efficient acceleration, before a cutoff sets in. For the subluminal shock cases, the spectra for mildly-relativistic shocks have spectral indices around 2.0-2.2. Highly-relativistic shocks have spectra as flat as $E_{\mathrm{p}}{ }^{-0.7}-E_{\mathrm{p}}{ }^{-1.5}$ at energies between $10^{9.5} \mathrm{GeV}$ and $10^{10.5} \mathrm{GeV}$. There is no universal spectral form, rather a variety of spectral shapes with a noticeable plateau-like structure developing at higher $\Gamma$ values. This structure is very probably related to the number of scattering cycles undergone by particles at a particular energy.

Our results can be summarised as follows:

1. Subluminal shock studies were made with a pitch scattering angle lying in the range $1 / \Gamma \leq \delta \theta \leq 10 / \Gamma$, approximately corresponding to a situation with a power spectrum of scattering waves, $P(k) / B^{2}=5 / 4 \sqrt{2} \cdot \Gamma^{-2} \cdot k^{-1}$ and with the neglect of cross-field diffusion. The resulting spectral slopes were roughly independent of inclination angle, though details of the features were different. A dependence of the spectral index $\alpha_{\mathrm{p}}$ on the shock boost factor $\Gamma$ was found, leading to spectra of $\alpha_{\mathrm{p}} \sim 2.0-2.1$ for mildly-relativistic shocks of $\Gamma \sim 10-30$, but producing much harder spectra $\left(0.7<\alpha_{\mathrm{p}}<\right.$ 1.5) for highly-relativistic shocks, $100<\Gamma<1000$.

The preceding paragraph implies that the particle spectra arising from relativistic shocks in GRB with very high boost factors between $100<\Gamma<1000$, have spectra flatter than $E_{\mathrm{p}}{ }^{-1.5}$, which is much flatter than AGN spectra where the dependence is $\sim E_{\mathrm{p}}{ }^{-2}$. Moreover, the above findings are supported by the work at lower $\Gamma$ of Niemec \& Ostrowski (2005) and Stecker et al. (2007). Observational evidence, see in Dingus (1995), regarding irregular and flat spectra from GRBs may be explained by the spectra we present. This work is also consistent with general observation of electron spectra that are injected at the terminal hotspots of lobes of the powerful FR-II radio galaxies which do not follow a single and universal power-law form, as shown in detail in Rudnick et al. (1994), Machalski et al. (2007), etc.

2. Superluminal shocks are only efficient in accelerating CRs up to $E_{\mathrm{p}} \sim 10^{5} \mathrm{GeV}$, resulting in spectral indices of $\alpha_{\mathrm{p}} \sim 2.0-2.3$. On the other hand, subluminal shocks are more 
efficient and able to accelerate CRs up to $E_{\mathrm{p}} \sim 10^{12} \mathrm{GeV}$, factors of $10^{9} \rightarrow 11$ above the particle injection energy.

3. We discussed the possible contributions of AGN and GRBs to the UHECR flux. For superluminal sources, such contributions can be excluded using current neutrino flux limits to normalise the spectrum. In the case of subluminal sources, the spectrum is normalised to the CR flux above the ankle, $E_{\text {min }}=10^{9.5} \mathrm{GeV}$. Using only AGN $(\Gamma=10)$, the spectrum fits very well the data, within experimental uncertainties. With a significant contribution from high relativistic shocks in GRBs $(100<\Gamma<1000)$, however, the total spectrum is too flat and it is difficult to explain the lower part of the spectrum around $E_{\mathrm{p}} \sim 10^{9.5} \mathrm{GeV}$. Even if UHECRs are accelerated in either external or internal shocks of GRBs, it is necessary to account for all the energy in accelerated particles, down to the injection energy. The total relativistic plasma output available from GRBs is only marginally sufficient to account for the total energy required in the extragalactic CR spectrum observed above the ankle. It therefore falls short of the requirement to supply all the energy implied by a low energy extrapolation of the observed, extra-galactic spectrum.

4. Recent Auger results indicate a correlation between $\mathrm{CR}$ arrival directions at the highest energies $\left(E_{\mathrm{p}}>\right.$ $\left.5.7 \times 10^{10} \mathrm{GeV}\right)$ and the distribution of AGN, see The Pierre Auger Collaboration (2007). This is the first significant evidence that the cosmic ray flux above the GZK cutoff originates from AGN predominantly in the supergalactic plane. The question of the origin of CRs below the GZK cutoff is not answered by this observation, but it is likely that more distant AGN contribute significantly to the flux, as AGN in the supergalactic plane make up the flux above the GZK cutoff. Moreover the output of X-ray active AGN within $60 \mathrm{Mpc}$ provides an energy density exceeding the local estimated total extra-galactic CR energy density by a factor $10^{4}$. It therefore seems reasonable to believe that the results from Auger can be explained by subluminal relativistic shock acceleration in AGN. It is now important to develop the simulation results further by including particle interactions, by making more detailed modelling of particle propagation in the inter-galactic medium and by investigating the source distribution of AGN in relation to observation in order to resolve the question of which AGN are the main sources of UHECRs.

5. Extragalactic, superluminal shocks are good candidates for the production of high energy neutrinos and photons. The energy density at low energies may be quite high compared to the observed flux of UHECRs, but hidden by galactic CRs. Neutrino- and photon fluxes may, on the other hand, be identified and are probably the only possibility to observe extragalactic, superluminal shocks.

Acknowledgements. We would like to thank the anonymous referee for the valuable corrections and comments which considerably improved this work and we are grateful to P. Biermann, F. Halzen and W. Rhode for extensive discussions on this work. The project was co-funded by the European Social Fund and National Resources (EPEAEK II) PYTHAGORAS, Greece.

\section{Appendix A: The simulation}

Our use of a Monte Carlo technique to solve the well known Boltzmann transport equation is dependent on the assumptions that the collisions represent diffusive scattering in pitch angle and that the scattering is elastic in the fluid frame where there is no residual electric field. We rely on a theoretical result that
Alfvén waves are limited to $V_{\mathrm{A}} \rightarrow c / \sqrt{3}$ to make the approximation that the scattering is elastic in the fluid frame. A phase averaged distribution function is appropriate to the diffusion approximation we employ which uses many small angle scatters. This approximation is abandoned for the highly anisotropic propagation encountered when particles traverse superluminal shocks.

We begin the simulation by injecting $10^{5}$ particles far upstream and of a weight $w_{\mathrm{p}}$ equal to 1.0. A splitting technique is used similar to the one used in the Monte Carlo simulations of Meli \& Quenby (2003a,b), so that when an energy level is reached such that only a few accelerated CRs remain, each particle is replaced by a number of $N$ particles of statistical weight $1 / N$. This makes it possible to follow a roughly constant number of CRs in reach decade of the accelerated energy scale. First order Fermi (diffusive) acceleration is then simulated by following the particles' guiding centres and allowing for numerous pitch angle scatterings in interaction with the assumed magnetised media, while at each shock crossing the particles gain an amount of energy determined by a transformation of reference frame.

Jones \& Ellison (1991)

review calculations made by themselves and others which demonstrate that in both parallel and oblique shocks an acceleration process related to repeated particle reflection at the shock is basic to the formation of the structure of collisionless shocks. Simulations and observation at the Earth's bow shock demonstrate the emergence of particles with several times the energy of particles in the upstream plasma. This significant energisation gives rise to important non-linear effects which modify the shock structure. Alternatively, we note that very large electrostatic potentials arise in the unipolar electrostatic field models for jets in AGN, see Gisler et al. (1989), and in GRB, see van Putten (2005). We may suppose that the bulk material is energised to some fraction of the full electrostatic potential while a small number of particles achieve significantly more energy to become the seed particles for further, diffusive shock acceleration. In this work, we simply assume the existence of a seed particle distribution such that a test particle approach is valid. A complete, many particle, two fluid model allowing acceleration to be followed over many decades of energy is yet to be realised. As a justification for a test particle approach, we note that Bell (1978a), Bell (1978b) and Jones \& Ellison (1991) have shown that "thin" sub-shocks appear even in the nonlinear regime, so at some energy above the plasma $\Gamma$ value, the accelerated particles may be dynamically unimportant while they re-cross the discontinuity. Another way of arriving at the test-particle regime is to inject particles well above the plasma particle energy when they are dynamically unimportant and thus require the seed particles to have already experienced preacceleration. Therefore, for our simulations the initial injection energy for the simulations is taken to be $\gamma \sim(\Gamma+100)$, when they are entered into the model upstream and directed towards the shock. The assumption of the pre-accelerated seed particles is also justified by the fact (indirectly inferred by observations), that in AGN jets, travelling shocks superimposed on the relativistic flows accelerated by pressure at the jet base, could provide the seed for the terminal "hot spot" acceleration. Moreover such seed particles appear in the neutron star binary merger scenario for GRBs as Narayan et al. (1992) showed, which includes the presence of pre-accelerated particles before any terminal shock acceleration phase.

The basic coordinate system employed to describe a shock is a Cartesian system $(x, y, z)$, where the shock plane lies on the $(y, z)$ plane. The reference frames used during the simulations 


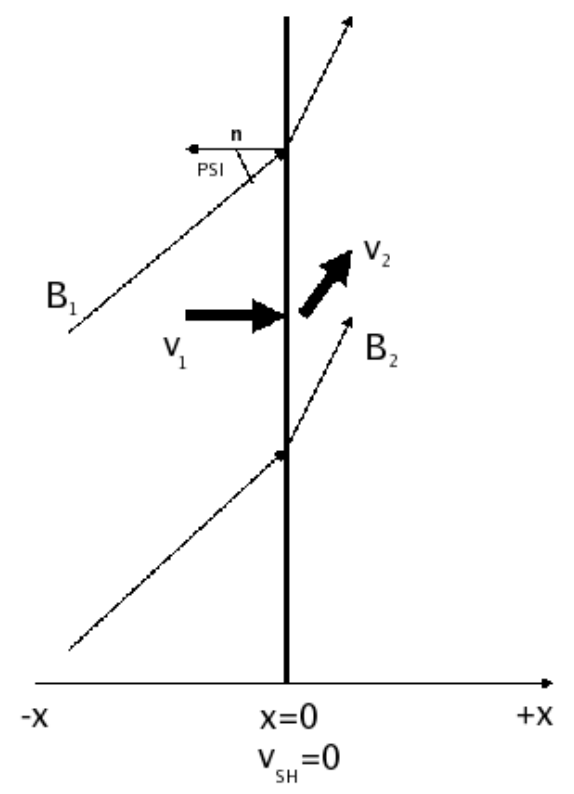

Fig. A.1. The coordinate system of a shock as seen in the so called (normal) shock frame.

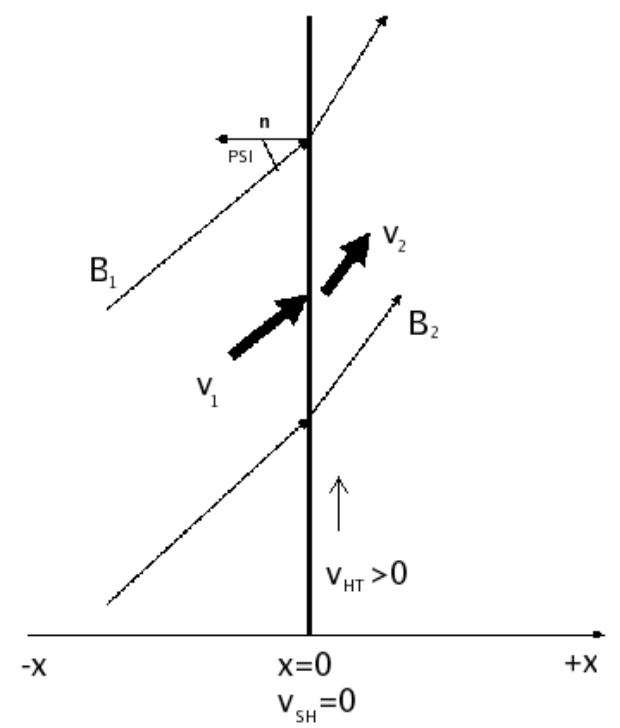

Fig. A.2. The coordinate system of a shock as seen in the so called de Hoffmann and Teller frame.

are the upstream and downstream fluid frames, the normal shock frame (NSH) and the de Hoffmann-Teller (HT) frame, see Figs. A.1 and A.2.

Standard theory assumes conservation of the first adiabatic invariant in the HT frame in order to determine reflection or transmission of the particles. Since in this frame the allowed and forbidden angles for transmission depend only on the input pitch and phase, not on rigidity, the results of previous, detailed investigation of this approximation, Hudson (1965), apply in our model. For an isotropic flux, the transmission coefficient $\zeta$ is simply assumed to be given by the particle flux conservation between an upstream magnetic flux tube area and the corresponding downstream flux tube area to which it connects

$\zeta=\frac{F B_{1}}{F B_{2}}$

where $F$ is the distribution function, Parker (1965). This expression is consistent with the conservation of the first adiabatic invariant in a gradual field increase from the upstream to the downstream field magnitude. Trajectory integration calculations, see Hudson (1965), giving the phase dependence of the probability of transmission as a function of phase and pitch angle, showed that the reflection percent plotted against pitch angle, never varied more than $20 \%$ from the mean value. Moreover, within this error range, the integrations were consistent with the flux conservation prediction based on Parker (1965) and the adiabatic invariant conservation. In the relativistic shock situation anisotropy renders the input to the shock from upstream, very anisotropic in pitch angle, but as discussed in Meli (2003), it is an acceptable approximation to randomise phase before transforming to the HT frame and then to use the adiabatic invariant to decide on reflection or transmission.

For the oblique shock cases studied here, provided the field directions encountered are reasonably isotropic in the shock frame, we know that $\tan \psi_{1}=\Gamma_{1}^{-1} \tan \psi_{\mathrm{NSH}} \sim \Gamma_{1}^{-1} \sim \psi_{1}$ where " 1 " and "NSH" refer to the upstream and normal shock frames respectively. The concentration of field vectors close to the $x$-axis in the upstream fluid frame allows a reasonable probability of finding a HT frame with a boost along the negative $y$-axis less than $c$. Making this boost then yields an upstream HT frame inclination, $\tan \psi_{\mathrm{HT}, 1}=\Gamma_{\mathrm{HT}, 1} \tan \psi_{1}$. While all particles are allowed to cross from downstream to upstream, only particles with a critical HT frame pitch angle, $\theta_{\mathrm{c}}$, given by

$\theta_{\mathrm{c}}=\arcsin \left(\frac{B_{\mathrm{HT}, 1}}{B_{\mathrm{HT}, 2}}\right)^{1 / 2}$

are allowed to cross upstream to downstream and conservation of the first adiabatic invariant is used to determine the new, downstream pitch angle. A compression ratio of 3 is used although some MHD conditions favour a value of 4 . Meli \& Quenby (2003b) do not find a great difference between the simulation results in these two cases. The results of Newman et al. (1992) suggest that it is legitimate to use Eq. (A.2) as a reasonable approximation. These authors checked the preservation of the first adiabatic invariant for the worst case, near perpendicular shock, employing trajectory integration with a realistic scattering field right up to the shock interface. At the critical angle for a shock with a compression ratio of 3 , deviations in the value of the adiabatic invariant were typically confined to within ten percent while larger effects tended to occur only towards 90 degree pitch angle. Particles are assigned a random phase so that a 3-dimensional transformation of momentum vectors can be achieved between the fluid and HT frames. Away from the shock, the guiding centre approximation is used so that a test particle moving a distance, $d$, along a field line at $\psi$ to the shock normal, in the plasma frame has a probability of collision within $d$ given by $P(d)=1-\exp (-d / \lambda)=R$, where the random number $R$ is $0 \leq R \leq 1$. Weighting the probability by the current in the field direction $\mu$ (i.e. $\cos \theta$ ) yields $d=-\lambda \mu \ln R$. The pitch angle is measured in the local fluid frame, while the value $x_{i}$ gives the distance of the particles to the shock front, where the shock is assumed to be placed at $x=0$. Furthermore, $x_{i}$ is defined in the shock rest frame and the model assumes variability in only one spatial dimension. Scattering in pitch is applied as described in the main body of this text.

In our simulations continuous Lorentz transformations are performed from the local plasma frames into the shock frame and back in order to check for crossing of the shock by the particle. All particles leave the system if they escape far downstream at the spatial boundary, $r_{\mathrm{b}}$. The downstream spatial boundary required can be estimated initially from the solution of the 
convection-diffusion equation in a non-relativistic, large-angle scattering approximation in the downstream plasma, which gives the chance of return to the shock as $\exp \left(u_{2} r_{\mathrm{b}} / \kappa_{\|}\right)$, where $u_{2}$ is the downstream plasma or convection velocity. In fact, we have performed many runs with different spatial boundaries to investigate the effect of the size of the acceleration region on the spectrum, so as to find a region where the spectrum is size independent. For computational economy, particles are also exited from the system if they reach a specified maximum energy $E_{\max }$.

If the shock is superluminal, so the physical picture of the shock drift acceleration applies, it is necessary to abandon the guiding centre approximation when the trajectories begin to intersect the shock surface. Instead, we consider the helical trajectory motion of each test-particle of momentum $p$, in the fluid frame, whether upstream or downstream, where the velocity coordinates $\left(v_{x}, v_{y}, v_{z}\right)$ of the particle are calculated in 3-dimensional space according to

$v_{x_{i}}=v_{i} \cos \theta_{i} \cos \psi_{i}-v_{i} \sin \theta_{i} \cos \phi_{i} \sin \psi_{i}$,

$v_{y_{i}}=v_{i} \cos \theta_{i} \sin \psi_{i}+v_{i} \sin \theta_{i} \cos \phi_{i} \cos \psi_{i}$

and

$v_{z_{i}}=-v_{i} \sin \theta_{i} \sin \phi_{i}$.

Here, $\theta_{i}$ is the pitch angle, $\phi_{i} \in(0,2 \pi)$ and $\psi_{i}$ is the angle between the magnetic field and the shock normal in the respective fluid frames $(i=1,2$ for upstream and downstream respectively).

We follow the trajectory in time, using $\phi_{i}=\phi_{\circ}+\omega t$, where $t$ is the time from detecting shock presence at $x_{\mathrm{NSH}}, y_{\mathrm{NSH}}, z_{\mathrm{NSH}}$ by using

$\mathrm{d} x=x_{\mathrm{NSH}}+v_{x_{i}} \delta t$,

$\mathrm{d} y=y_{\mathrm{NSH}}+v_{y_{i}} \delta t$

and

$\mathrm{d} z=z_{\mathrm{NSH}}+v_{z_{i}} \delta t$

assuming that $\delta t=r_{g} / H c$ where $H \geq 100$ and $r_{g}$ is the Larmor radius. The particle's gyrofrequency $\omega$ is given by the relation, $\omega_{i}=e\left|\boldsymbol{B}_{i}\right| / \gamma_{i}, \boldsymbol{B}_{i}$ is the magnetic field, $\gamma_{i}$ is the particle's boost factor and $e$ is its charge in gaussian units.

We follow the helical trajectory of each particle in time $t$, in the new frame where $t$ is the time from detecting the shock intersection at $(x, y, z)$ until the trajectory has performed one gyro period without re-intersecting the shock surface. Nevertheless, because of the peculiar properties of the helix we need to establish where a particle, starting off in the upstream frame, with a particular $\theta$ and $\phi$ first encounters the shock. To establish when the shock encounter happens, we choose to go back a whole period, $T_{i}=2 \pi / \omega_{i}$ by reversing signs of the helix velocity coordinates and by keep checking throughout the simulation to determine if the particle trajectory encounters the shock front, placed at $x=0$ in the shock rest frame. If the particle encounters the shock then the suitable Lorentz transformation to the relevant fluid rest frame is made and we continue following the particle helical trajectory until shock intersections cease. At this juncture, the guiding centre is followed in the same way as in the diffusive acceleration picture of the subluminal shocks. During the helical phase of the numerical integration, the prescription for pitch angle scatter is applied as in the general plasma frame motions, in order to more realistically simulate a mean plus chaotic field situation, where turbulence is clearly present close to a shock.
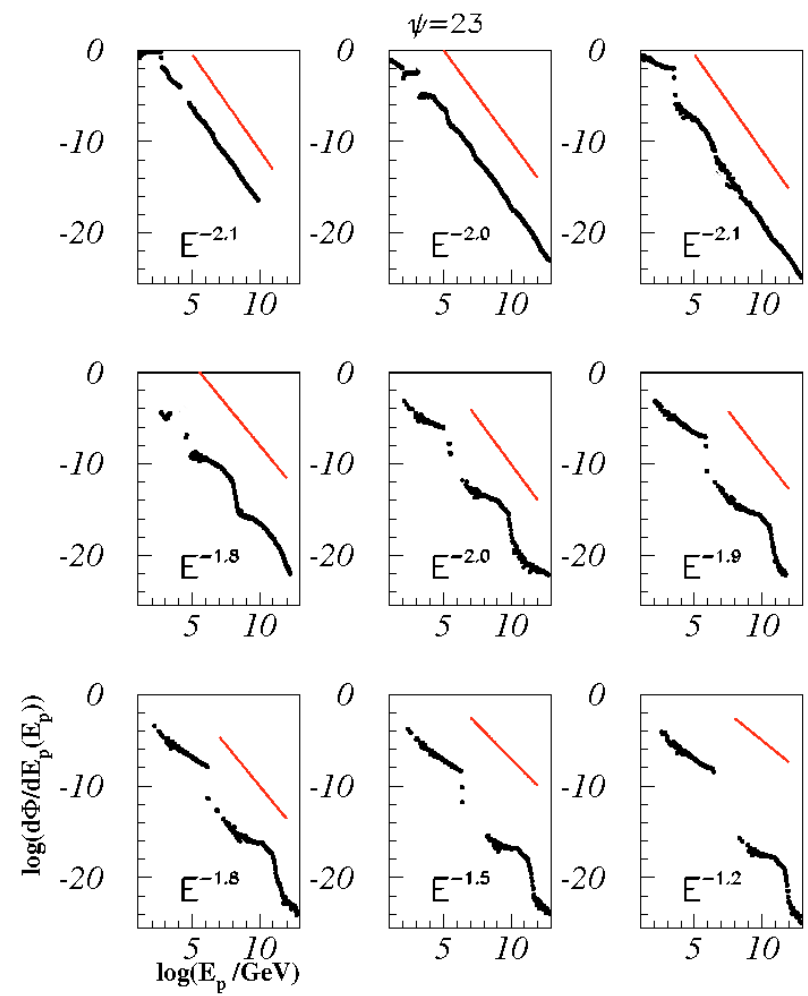

Fig. B.1. Subluminal spectra for $\psi=23^{\circ}$ and different $\Gamma$ : $\Gamma=10,20,30$ is displayed in the first row, in the middle, $\Gamma=100,300,500$ is shown and $\Gamma=700,900,1000$ is the bottom row. The black dots in each graph represent the simulation result. The straight line shows the single powerlaw for comparison. The spectral behaviour is indicated in the lower left corner of each graph.

\section{Appendix B: The spectra}

The particle spectra resulting from the simulation are presented in Figs. B.1-B.3. In each of the figures, the simulated particle spectra are shown (black dots) for three different shock angles, $\psi=23^{\circ}, 33^{\circ}, 43^{\circ}$, for nine different boost factors, $\Gamma=10,20,30$ in the first row, starting from the left, $\Gamma=100,300,500$ in the middle row and $\Gamma=700,900,1000$ in the lower row. Each graph shows the logarithm of the proton spectrum $\mathrm{d} \Phi / \mathrm{d} E_{\mathrm{p}}$ in arbitrary units versus the proton's energy in units of GeV. Note that the spectrum can be expressed more generally in terms of the particle's boost factor $\gamma=E_{\mathrm{p}} /\left(m_{\mathrm{p}} c^{2}\right)$. Therefore, the results are also valid for nuclei with higher mass (e.g. Fe). In the present investigation protons are considered.

Figures B.1-B.3 show that the spectral shape starts to deviate from a power-law as $\Gamma$ increases with the onset of plateau formation. This may be understood since the particles are swept away rapidly downstream with a low chance of return upstream for high $\Gamma$ factors. Typically only $20 \%$ of the particles return to the shock after one shock cycle. This small chance of return to the shock, except for a relatively small subset of "histories" of downstream pitch angle particle behaviour, produces the spectral irregularities and the anisotropy seen for these returning particles. We note that in the case of $\Gamma=10-30$ relatively smooth spectra are produced, but spectra become more structured (plateau-like) at the more extreme values $\Gamma \rightarrow 1000$. In the latter cases the effects of individual acceleration cycles are clearly evident. The mechanism of plateau development as an acceleration cycle effect is implicit in Figs. 6 of Protheroe (2001) and 2 of Stecker et al. (2007) and seems to be independent 

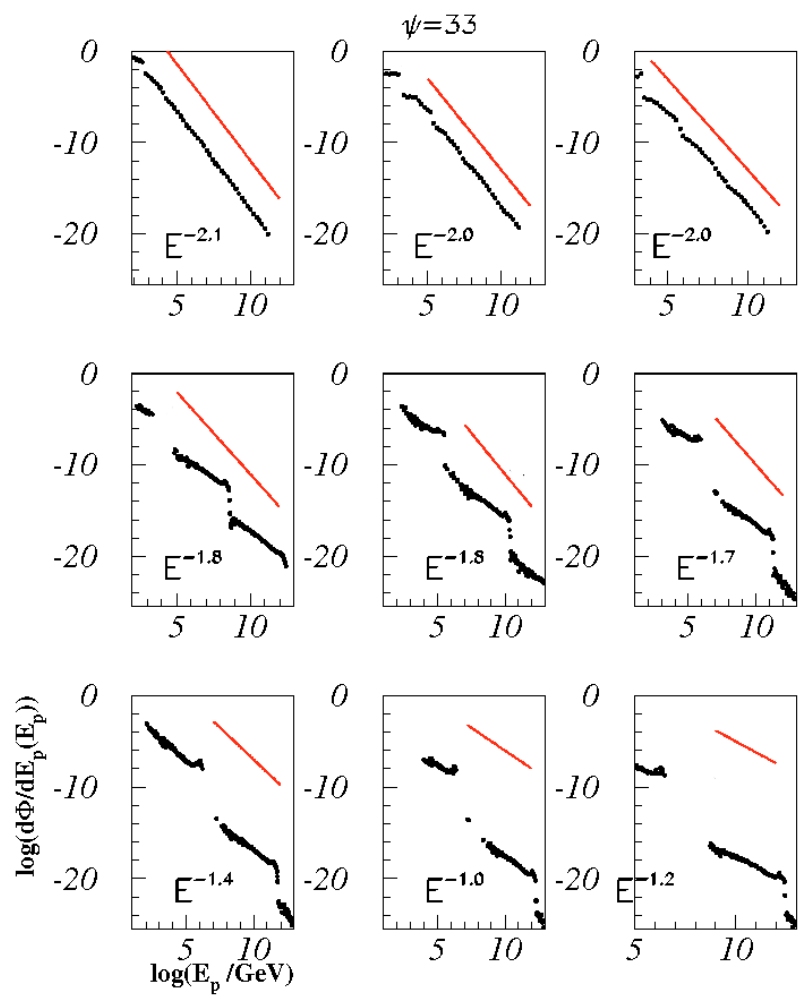

Fig. B.2. Subluminal spectra for $\psi=33^{\circ}$ and different $\Gamma$, as in Fig. B.1.

of the shock inclination angle for a particular scattering model. The structured spectra can also be seen in the lower $\Gamma$ large angle scatter model simulations of Quenby \& Lieu (1989) and Ellison et al. (1990) while Protheroe (2001) shows a similar contrast in behaviour, between large and small angle scattering models up to $\Gamma=20$.

The initial, low energy bump found in most of the spectra is due to the monochromatic energy injection of the particles. We approximate the spectra with a power-law (straight lines seen in the figures) at higher energies and thus artificial injection features are excluded from the subsequent calculations. The maximum energy is chosen to be $10^{21} \mathrm{eV}$ as required by the discussion of Sect. 1.2. The plateau-like parts in the spectra at higher energies are physical features, especially at high boost factors, as particles continue to be accelerated in a second cycle. The lower energy part of the spectrum is dominated by particles undergoing one acceleration shock cycle, while the second bump in the spectrum represents particles having completed two cycles. A shock cycle is a shock crossing from upstream to downstream to upstream. Since some upstream particles can suffer repeated reflection before downstream transmission, this effect increases the statistical energy gain of the particles in their overall encounter with the shock surface.

The aim of fitting a single power-law to the computed spectral points is primarily to examine the variation of primary spectra with the $\Gamma$ factor of the shock. The spectral points themselves are difficult to compare because the structure becomes more complex with the increasing boost factor. As discussed in the main text, the relevant way to compare the particle spectra to the data is to fit the energy range observed in cosmic rays. However, the straight lines in Figs. B.1-B.3 together with the values written in the lower left corner of each graph indicate the single power-law fit over the entire energy range and are shown for comparison between each case.
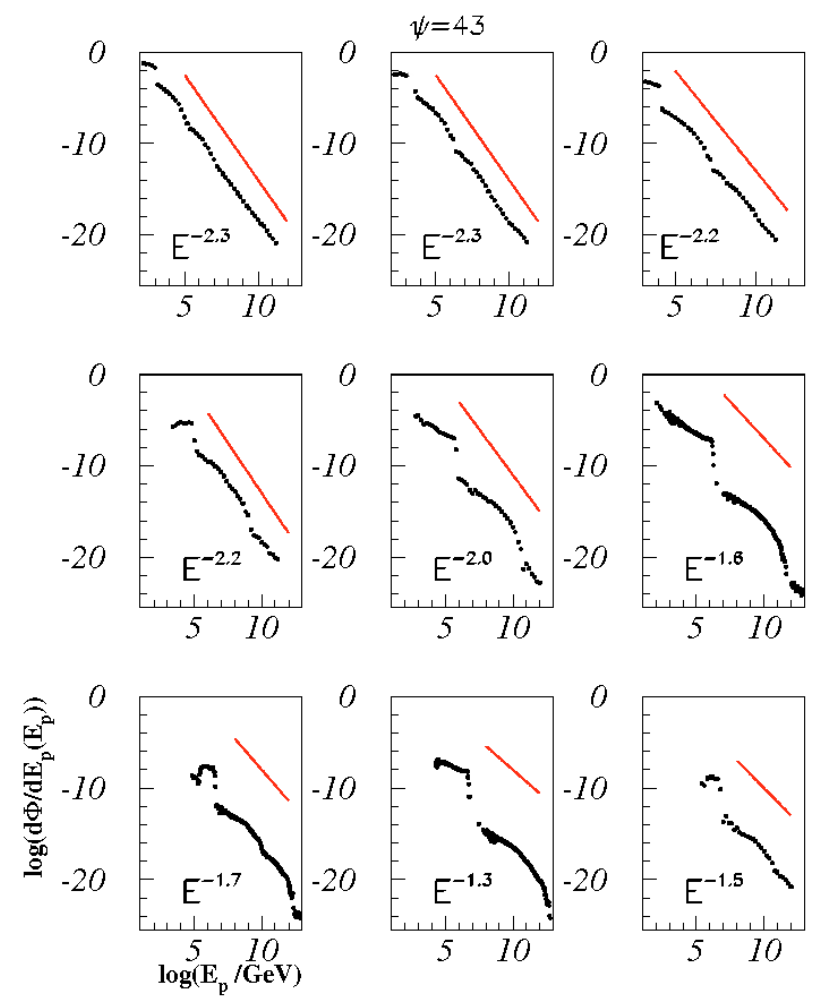

Fig. B.3. Subluminal spectra for $\psi=43^{\circ}$ and different $\Gamma$, as in Fig. B.1.

Table B.1. Spectral indices for a single power-law comparison (fit over the entire energy range) for subluminal shocks of different boost factors and three inclination angles.

\begin{tabular}{l|ccc}
\hline \hline$\Gamma$ & $\alpha_{\mathrm{p}}\left(\psi=23^{\circ}\right)$ & $\alpha_{\mathrm{p}}\left(\psi=33^{\circ}\right)$ & $\alpha_{\mathrm{p}}\left(\psi=43^{\circ}\right)$ \\
\hline 10 & 2.1 & 2.1 & 2.3 \\
20 & 2.0 & 2.0 & 2.3 \\
30 & 2.1 & 2.0 & 2.2 \\
100 & 1.8 & 1.8 & 2.2 \\
300 & 2.0 & 1.8 & 2.0 \\
500 & 1.9 & 1.7 & 1.6 \\
700 & 1.8 & 1.4 & 1.7 \\
900 & 1.5 & 1.0 & 1.3 \\
1000 & 1.2 & 1.2 & 1.5 \\
\hline
\end{tabular}

Table B.1, based on the above fits, shows the dependence of the spectral index on the boost factor for the three shock inclination angles $\psi=23^{\circ}, 33^{\circ}, 43^{\circ}$. For each of the three angles, the spectra become harder with the increasing $\Gamma$ factor of the shock. Due to the structure in the spectra, these values represent simple first order approximations which are useful for comparison of the simulation results with the data. CR data include large statistical and systematic errors, which would make it difficult to distinguish features attributable to the acceleration mechanism other than single or broken power-laws.

The computed spectral flattening with increasing $\Gamma$ shows that particle spectra, arising from relativistic shocks with boost factors between $100<\Gamma<1000$, have spectral indices ranging between $\alpha_{\mathrm{p}} \sim 2.1-1.5$. Some values of the indices found in Table B.1. are even lower. The relativistic flattening effect is consistent with the work of Baring (1999), Baring (2004), Stecker et al. (2007). In contrast, shocks with $\Gamma \sim 10-30$ yield spectral indices with values between $2.0<\alpha_{\mathrm{p}}<2.3$.

Based upon the figures quoted in Table B.1, it can be seen that single power-law fits at a particular $\Gamma$ give comparable 
values for different angles $\psi$. Simulation test runs performed using a more extensive series of values of $\psi$ at a selected $\Gamma$ followed the same trend as long as the scattering model was fixed. The present results deviate from earlier investigations by Kirk et al. (2000), Ostrowski \& Bednarz (2002), which showed a saturation of the flattening of the spectra with the boost factor, such that $\alpha_{\mathrm{p}} \rightarrow 2.33$ for $\Gamma \rightarrow \infty$. However, these latter studies were concerned with the special case of extremely low values for the magnitude of the pitch angle change for each scattering encounter.

\section{References}

Achterberg, A., Ackermann, M., Adams, J., et al. 2007, Phys. Rev. D, 76, 042008 Aharonian, F., Akhperjanian, A. G., Barrio, J. A., et al. 1999, Phys. Rev. D, 59, 092003

Ahlers, M., Anchordoqui, L. A., Goldberg, H., et al. 2005, Phys. Rev. D, 72, 023001

Akhieser, A. I., Akhieser, I. A., Polovin, R. V., Sitenko, A. G., \& Stepanov, K. N. 1975, Plasma Electrodynamics (New York: Pergamon Press)

Antoni, T., Apel, W. D., Badea, A. F., et al. 2005, Astropart. Phys., 24, 1

Antonov, R. A., Anokhina, A. M., Galkin, V. I., et al. 1995, Astropart. Phys., 3, 231

Asakimori, K., Burnett, H. T., et al. 1995, Int. Cosmic Ray Conf., 3., 707

Ave, M., Hinton, J. A., Knapp, J., Marchesini, M., \& Watson, A. A. 2001, Int. Cosmic Ray Conf., 381

Baring, M. 1999, Int. Cosmic Ray Conf., 4, 5

Baring, M. G. 2004, BAAS, 36, 942

Becker, J. K. 2008, Phys. Rep., 458, 173

Becker, J. K., \& Biermann, P. L. 2008 [ArXiv: 0805. 1498]

Becker, J. K., Biermann, P. L., \& Rhode, W. 2005, Astropart. Phys., 23, 355

Becker, J. K., Stamatikos, M., Halzen, F., \& Rhode, W. 2006, Astropart. Phys., 25,118

Bednarz, J., \& Ostrowski, M. 1998, Phys. Rev. Lett., 80, 3911

Begelman, M. C., \& Kirk, J. G. 1990, ApJ, 353, 66

Bell, A. R. 1978a, MNRAS, 182, 147

Bell, A. R. 1978b, MNRAS, 182, 443

Biermann, P. L., \& Strittmatter, P. A. 1987, ApJ, 322, 643

de Hoffmann, F., \& Teller, E. 1950, Phys. Rev., 80, 692

Dieckmann, M. E., Meli, A., Shukla, P. K., Drury, L. O. C., \& Mastichiadis, A. 2008, Plasma Phys. \& Controlled fusion, 50, 6

Dingus, B. L. 1995, Astrophys. Space Sci., 231, 187

Ellison, D. C., \& Double, G. P. 2004, Astropart. Phys., 22, 323

Ellison, D. C., Reynolds, S. P., \& Jones, F. C. 1990, ApJ, 360, 702

Falcke, H., Malkan, M. A., \& Biermann, P. L. 1995, A\&A, 298, 375

Fermi, E. 1949, Phys. Rev., 75, 1169

Fermi, E. 1954, ApJ, 119, 1

Forman, M. A. 1970, Plan. Space Sci., 18, 25

Gallant, Y. A., Achterberg, A., \& Kirk, J. G. 1999, A\&AS, 138, 549

Gehrels, N., \& Michelson, P. 1999, Astropart. Phys., 11, 277

Gisler, G. R., Lovelace, V. E., \& Norman, M. I. 1989, A\&A, 342, 135

Glasmacher, M. A. K., Catanese, M. A., Chantell, M. C., et al. 1999, Astropart. Phys., 10, 291

González, M. M., Dingus, B. L., Kaneko, Y., et al. 2003, Nature, 424, 749

Grigorov, N. L., Savenko, I. A., Basilova, R. N., et al. 1975, in Cosmic Rays, ed. S. N. Vernov, \& L. I. Dorman, 227

Guetta, D., Hooper, D., Alvarez-Mun Iz, J., Halzen, F., \& Reuveni, E. 2004, Astropart. Phys., 20, 429

Halzen, F., \& Hooper, D. 2002, Rep. Progr. Phys., 65, 1025

Hasinger, G., Miyaji, T., \& Schmidt, M. 2005, A\&A, 441, 417

Hillas, A. M. 1984, ARA\&A, 22, 425

Hudson, P. D. 1965, MNRAS, 131, 23
Hurley, K., Dingus, B. L., Mukherjee, R., et al. 1994, Nature, 372, 652

Jokipii, J. R. 1987, ApJ, 313, 842

Jones, F. C., \& Ellison, D. C. 1991, Space Sci. Rev., 58, 259

Kennel, C. F., \& Petscheck, H. E. 1966, J. Geophys. Res., 71, 1

Khristiansen, G. B., Fomin, Yu. A., Kalmykov, N. N., et al. 1994, Astropart. Phys., 2, 127

Kirk, J. G., Guthmann, A. W., Gallant, Y. A., \& Achterberg, A. 2000, ApJ, 542, 235

Krasilnikov, D. D., Knurenko, S. P., Krasilnikov, A. D., et al. 1985, Int. Cosmic Ray Conf., 194

Krymskii, G. F. 1977, Akademiia Nauk SSSR Doklady, 234, 1306

Liang, R. A. 1981, MNRAS, 195, 261

Laing, R. A., \& Brindle, A. H. 2001, Particles and Fields in Radio Galaxies, ASP Conf. Ser., 250

Lerche, I., \& Schramm, D. N. 1977, ApJ, 216, 881

Mc Carthy, P. J., van Breugel, W., Spinrad, H., \& Djorgovski, S. 1987, A\&A, $321 \mathrm{~L}, 29$

Machalski, J., Chyży, K. T., Stawarz, Ł., \& Kozieł, D. 2007, A\&A, 462, 43

Mannheim, K. 1995, Astropart. Phys., 3, 295

Mannheim, K., Protheroe, R. J., \& Rachen, J. P. 2001, Phys. Rev. D, 63, 23003

Medvedev, M. V. 1999, Phys. Plasmas, 6, 2191

Meli, A. 2003, Ph.D. Thesis, Imperial College of Science, Technology and Medicine, London, UK

Meli, A., \& Quenby, J. J. 2003a, Astropart. Phys., 19, 637

Meli, A., \& Quenby, J. J. 2003b, Astropart. Phys., 19, 649

Moran, E. C., Kay, L. E., Davis, M., Filippenko, A. V., \& Barth, A. J. 2001, ApJ, $556, \mathrm{~L} 75$

Murase, K., \& Nagataki, S. 2006, Phys. Rev. D, 73, 063002

Narayan, R., Paczynski, B., \& Piran, T. 1992, A\&A, 395, L83

Newman, P. L., Moussas, X., Quenby, J. J., Valdes-Galicia, J. F., \& Theodossiou-Ekaterinidi, Z. 1992, A\&A, 255, 443

Niemec, J., \& Ostrowski, M. 2005, Geophysical Monograph, 156, AGU, Washington, DC, 59

Niemec, J., \& Ostrowski, M. 2007, Int. Cosmic Ray Conf. [arXiv: 0705.4453]

Ostrowski, M., \& Bednarz, J. 2002, A\&A, 394, 1141

Ozawa, S., Tibet Asgamma Coll., et al. 2003, Int. Cosmic Ray Conf., 143

Parker, E. N. 1958, Phys. Rev., 109, 1328

Parker, E. N. 1965, ApJ, 142, 1086

Piran, T. 1999, Phys. Rep., 314, 575

Protheroe, R. J. 2001, Int. Cosmic Ray Conf., 6, 2006

Pugliese, G., Falcke, H., Wang, Y. P., \& Biermann, P. L. 2000, A\&A, 358, 409

Quenby, J. J., \& Lieu, R. 1989, Nature, 342, 654

Quenby, J. J., \& Meli, A. 2005, Geophysical Monograph, 156, AGU, Washington, DC, 9

Rudnick, L., Katz-Stone, D. M., \& Anderson, M. C. 1994, ApJS, 90, 955

Schneid, E. J., Bertsch, D. L., Fichtel, C. E., et al. 1992, A\&A, 255, L13

Sreekumar, P., Bertsch, D. L., Dingus, B. L., et al. 1998, ApJ, 494, 523

Stecker, F. W. 2005, Phys. Rev. D, 72, 107301

Stecker, F. W., Baring, M. G., \& Summerlin, E. J. 2007 [arXiv: 0707.4676]

The High Resolution Fly's Eye Collaboration 2002 [arXiv: astro-ph/0208301]

The Pierre Auger Collaboration 2007, Science J., 318, 939

van Putten, M. H. P. M. 1997, Phys. Rev. D, 55, 4705

van Putten, M. H. P. M. 2005, Gravitational Radiation, Luminous Black Holes and Gamma-Ray Burst Supernovae (Cambridge University Press)

Vietri, M. 1995, ApJ, 453, 883

Vietri, M., De Marco, D., \& Guetta, D. 2003, ApJ, 592, 378

Waxman, E. 2000, ApJS, 127, 519

Waxman, E., \& Bahcall, J. N. 1997, Phys. Rev. Lett., 78, 2292

Waxman, E., \& Bahcall, J. N. 1999, Phys. Rev. D, 59, 23002

Webb, G. M. 1987, ApJ, 319, 215

Yamamoto, T., et al. 2007, Int. Cosmic Ray Conf., 7, 387

Yoshida, S., Hayashida, N., Honda, K., et al. 1995, Astropart. Phys., 3, 105 